\title{
Corpus
}

$3 \mid 2004$

Usage des corpus en phonologie

\section{Elaboration d'un corpus morphophonologique : l'épenthèse consonantique à la frontière suffixale en français}

Claudine Pagliano

\section{(2) OpenEdition}

Journals

Édition électronique

URL : http://journals.openedition.org/corpus/257

DOI : $10.4000 /$ corpus.257

ISSN : 1765-3126

Éditeur

Bases; corpus et langage - UMR 6039

Édition imprimée

Date de publication : 1 décembre 2004

ISSN : 1638-9808

Référence électronique

Claudine Pagliano, «Elaboration d'un corpus morphophonologique : l'épenthèse consonantique à la frontière suffixale en français », Corpus [En ligne], 3 | 2004, mis en ligne le 02 décembre 2005, consulté le 07 septembre 2020. URL : http://journals.openedition.org/corpus/257 ; DOI : https://doi.org/ 10.4000 /corpus. 257 


\title{
Elaboration d'un corpus morphophonologique : l'épenthèse consonantique à la frontière suffixale en français
}

\author{
Claudine PAGLIANO \\ MoDyCo, UMR 7114, Paris 10
}

Résumé : Cet article présente les différentes étapes nécessaires à la construction d'un corpus visant l'étude des épenthèses consonantiques entre radicaux et suffixes en français, depuis le rassemblement des données brutes jusqu'au corpus final, débarrassé des termes dont la consonne candidate s'est avérée ne pas être épenthétique. Ce faisant, il montre les limites d'une extraction automatique des termes dérivés dans ce cadre morphophonologique. Enfin, il souligne le rôle d'un corpus raisonné dans la progression de l'analyse et la nécessaire interaction entre empirie et théorie.

\section{Introduction}

Cet article présente les différentes étapes de l'élaboration d'un corpus propre à l'étude de l'épenthèse consonantique à la frontière dérivationnelle suffixale en français, et se propose également de mettre en lumière le rôle de ce corpus dans l'analyse.

Lors de la dérivation suffixale en français apparaissent parfois des consonnes épenthétiques. Ainsi bijoutier, formé à partir de la base bijou et du suffixe -ier qui figure par exemple dans quincaillier ou charcutier, présente-t-il un [t] qui n'appartient à aucun de ses éléments constitutifs. Cependant, tous les dérivés ne présentent pas d'élément épenthétique à la frontière suffixale : bleuâtre par exemple est formé à partir de la base bleu et du suffixe -âtre sans qu'aucune consonne ne soit insérée. Il s'agit donc d'élaborer un corpus qui permette d'établir la distribution des épenthèses consonantiques dans cette position morphologique particulière, ce qui implique de 


\section{PAgLiano}

recenser les unités lexicales construites présentant une consonne épenthétique entre un radical et un suffixe en français. De l'examen systématique des contextes d'apparition d'une épenthèse naîtra une hypothèse quant à l'identité phonologique de la frontière morphologique.

Dans un premier temps, je présenterai les modalités qui ont présidé au rassemblement des termes dérivés, termes qui sont susceptibles d'entrer dans le cadre de l'étude envisagée. Ce faisant, je soulignerai la place que tiennent les options théoriques retenues ${ }^{1}$ lors de cette première étape. Je me tournerai ensuite vers la double procédure de filtre qui a permis de passer d'une base de données brute à un corpus raisonné, propre à étayer l'étude envisagée. La troisième partie mettra en évidence la contribution du corpus constitué dans la démarche analytique liée à la problématique de l'épenthèse consonantique à la frontière suffixale.

\section{Constitution d'une base de données brute}

La première étape de la constitution d'un corpus est la délimitation de l'objet d'étude. Il faut donc définir au préalable les dimensions temporelle, sociale et géographique retenues, ainsi que le type de données - orales ou écrites - envisagées.

\subsection{Circonscription des données}

Toute décision intempestive dans ces différents domaines pourrait biaiser l'analyse. Comment établir en effet une période favorite de création des unités lexicales retenues, sans connaître l'importance éventuelle du moment d'apparition d'un terme dans le processus d'épenthétisation ? Autrement dit, peut-on décider arbitrairement que la consonne épenthétique [d] présente dans faisandeau (faisan-d-eau), attesté dès 1393, n'est pas due au même processus d'insertion que le [z] du néologisme faucuseté (faux cul-z-eté) ? Pour cette même raison de manque d'information quant aux paramètres pertinents, le corpus doit

1. Cet article présente le corpus élaboré dans le cadre d'une thèse doctorale soutenue en décembre 2003 à l'Université de Nice Sophia-Antipolis (Pagliano 2003). 

Elaboration d'un corpus morphophonologique : l'épenthèse
consonantique

comporter aussi bien les termes figurant à l'écrit que ceux relevés uniquement à l'oral.

Se limiter à la langue "standard » pose un double problème, celui de l'identification de ce "standard" tout d'abord, celui de sa délimitation ensuite, en termes de niveaux de langue comme de langues de spécialité. Le «français standard ", à supposer qu'une telle entité existe, constitue un ensemble dont les frontières ne sont pas nettes (cf. Pagliano 2003 : 302-309 pour une présentation de diverses acceptions du concept), ce qui interdit toute affirmation trop véhémente quant à l'appartenance d'un corpus à cette langue standard et à elle uniquement. De plus, le concept est lié à la notion de langue " correcte ", or nombre de termes présentant des épenthèses consonantiques, en tant que néologismes ne figurent pas dans les dictionnaires : comment juger de leur degré d'acceptabilité par la langue standard ? Par ailleurs, cette étude ne souhaite en aucune façon se réduire à un français artificiel ; elle a, au contraire, pour ambition de s'intéresser aux mécanismes réels de la langue, c'est pourquoi la prise en compte de la variation y est nécessaire, excluant par là-même toute référence à un système unique. Comment jauger en outre l'influence réciproque de termes du vocabulaire " scientifique » et de termes de la langue "standard » en ce qui concerne ce phénomène de l'épenthèse consonantique?

Enfin, le même raisonnement empêche d'exclure du corpus des termes qui semblent relever de français régionaux. Il est vrai que ces variétés de français exploitent des mécanismes de formation qui peuvent être différents de ceux de «la» langue française. Cependant, rien ne permet de déterminer, hors de l'analyse d'un corpus, qu'une épenthèse dans une unité lexicale donnée est le résultat d'un mécanisme dialectal plutôt que d'un processus typiquement français.

Antérieurement à l'analyse du corpus donc, rien ne permet d'établir les critères pertinents, qu'ils relèvent de la variation sociale, régionale, stylistique ou diachronique. C'est pourquoi aucun terme candidat n'est exclu d'emblée. 


\section{PAgLiano}

\subsection{Exploitation automatique d'une liste de termes}

Dans cette optique de recherche de contexte déclencheur, le corpus doit pouvoir prétendre, sinon à l'exhaustivité - impossible du fait de l'évolution permanente de la langue -, du moins à une forte représentativité. C'est pourquoi a été envisagée dans un premier temps une extraction automatique des unités lexicales porteuses d'épenthèses dans la position souhaitée, à partir d'une liste informatisée de 64.296 termes. Il est à noter que des sources comme Internet, Frantext ou les journaux informatisés n'ont pas pu être exploitées dans le cadre de cette constitution de corpus, principalement faute de logiciels permettant d'interroger ces bases en fonction des suffixes et des radicaux en jeu dans la dérivation ; s'ajoutent à cela le "bruit» généré par des fautes de frappe ou de découpage de la chaîne, et le manque de contrôle quant à la réalisation des termes par un locuteur natif. Ce type de sources ne semble exploitable avec profit que dans le cadre de l'étude d'un suffixe donné (cf. Plénat \& al. 2002), mais non pour l'ensemble de la dérivation du français.

La base initiale de la source de mots construits contenant des épenthèses est une liste informatisée de 64.296 mots français établie par le laboratoire «Langues, textes, traitement informatique, cognition » (Lattice, UMR 8094) pour les besoins du traitement automatique des langues, modifiée par quelques ajouts et troncations personnels, lorsque certains termes se sont révélés inattestés (cachoutanique par exemple) ou manifestement le produit d'erreurs de traitement.

L'idée qui sous-tend la méthode d'extraction des unités lexicales comprenant une épenthèse est la suivante : si une unité lexicale comporte une épenthèse entre le radical et le suffixe, cet élément consonantique n'appartient $\mathrm{ni}$ au radical ni au suffixe. La démarche envisagée consiste dans un premier temps à écarter les préfixes puis les accents graphiques et les cédilles. Ensuite, les formes ainsi obtenues sont comparées automatiquement entre elles, en commençant par la gauche pour isoler les thèmes, puis par la droite pour déceler les suffixes en reprenant ces formes une deuxième fois. Les séquences sont 
Elaboration d'un corpus morphophonologique : l'épenthèse consonantique

comparées en commençant par la plus longue possible, de façon à obtenir des thèmes et non une liste alphabétique. Il s'agit là d'un traitement purement mécanique, portant sur des chaînes de caractères, et non linguistique : l'objectif est d'obtenir une première récolte qui se base uniquement sur la comparaison des unités lexicales entre elles et non sur une liste de suffixes préalablement établie, qui ne serait pas complètement objective puisqu'elle constituerait nécessairement le fruit d'une analyse antérieure.

Les exemples suivants permettent d'illustrer le fonctionnement du programme d'extraction :

\begin{tabular}{|l|l|l|l|l|l|l|l|l|}
\hline Etapes & \multicolumn{7}{|c|}{ Exemples } \\
\hline $\begin{array}{l}\text { Corpus } \\
\text { brut }\end{array}$ & forêt & $\begin{array}{l}\text { déforesta- } \\
\text { tion }\end{array}$ & glace & $\begin{array}{l}\text { dégla- } \\
\text { çage }\end{array}$ & $\begin{array}{l}\text { remor- } \\
\text { quage }\end{array}$ & remorque & déglutir & $\begin{array}{l}\text { dégluti } \\
\text {-tion }\end{array}$ \\
\hline $\begin{array}{l}\text { élision des } \\
\text { préfixes }\end{array}$ & forêt & forestation & glace & glaçage & $\begin{array}{l}\text { remor- } \\
\text { quage }\end{array}$ & remorque & déglutir & $\begin{array}{l}\text { dégluti- } \\
\text { tion }\end{array}$ \\
\hline $\begin{array}{l}\text { traitement } \\
\text { des } \\
\text { accents et } \\
\text { du ç }\end{array}$ & forest & forestation & glace & glacage & $\begin{array}{l}\text { remor- } \\
\text { quage }\end{array}$ & remorque & deglutir & $\begin{array}{l}\text { degluti } \\
\text {-tion }\end{array}$ \\
\hline $\begin{array}{l}\text { comparai- } \\
\text { son par la } \\
\text { gauche }\end{array}$ & $\varnothing$ & ation & $\mathrm{e}$ & age & age & $\mathrm{e}$ & r & tion \\
\hline $\begin{array}{l}\text { comparai- } \\
\text { son par la } \\
\text { droite }\end{array}$ & $\varnothing$ & a & $\varnothing$ & $\varnothing$ & $\varnothing$ & $\varnothing$ & r & $\varnothing$ \\
\hline
\end{tabular}

La première étape consiste à ôter les préfixes. Dans le mini-corpus proposé, six séquences comportent à l'initiale une suite de lettres pouvant correspondre à un préfixe : déforestation, déglaçage, remorquage, remorque, déglutir, déglutition. Pourtant, seules deux d'entre elles vont se retrouver amputées, un traitement manuel permettant de "sauver» remorque et remorquage d'une part, déglutir et déglutition d'autre part: *morque et *glutir ne sont ni des unités lexicales attestées en français, ni même possibles par commutation avec d'autres unités si l'on tient compte du signifié. 


\section{PAgLiano}

L'étape suivante, à savoir le traitement des accents, permet d'une part de convertir le $\hat{e}$ de forêt en la séquence -es-, d'autre part d'ôter les accents des « faux préfixes » de déglutir et déglutition. On obtient alors respectivement forest, deglutir et deglutition. Le traitement du ç constitue la dernière étape de préparation préalable du corpus ; seul glaçage est concerné et se voit transformé en glacage.

Les étapes de comparaison sont celles que l'on trouve dans le programme " Epenthèse », réalisé par Yvon Deschamps, maître de conférences de la faculté d'Orsay pour les besoins de cette étude. Il s'agit de la comparaison des éléments, par la gauche d'abord, puis par la droite de ce qui reste. Forêt, réécrit forest, et forestation permettent d'isoler la séquence -ation. Glace et glacage, anciennement glaçage, détachent -age d'un côté et -e de l'autre, de même que remorquage et remorquer. La comparaison de déglutir et déglutition met quant à elle en évidence les terminaisons $-r$ et -tion.

La dernière étape du processus consiste à comparer ces terminaisons en commençant par la séquence la plus longue et par la droite. Si l'on ne commençait pas par la séquence la plus longue mais la plus courte, on obtiendrait -ag- pour -age comparé avec - $e$, ce qui serait une aberration au regard de l'objectif poursuivi. En commençant par la séquence la plus longue sont isolés - $a$ - à partir de -ation et -tion, puis $-r$ puisque ce mini-corpus n'en comporte pas d'autre.

Cette méthode de travail a rencontré dans les faits plusieurs difficultés qui ont conduit à une marginalisation des données issues de ce traitement automatique dans la base de données finale.

\subsection{Orientation vers un traitement partiellement manuel des sources écrites}

Une première complication tient aux alternances de radicaux. Certaines obéissent à des règles, ce qui permet au locuteur d'identifier les radicaux comme étant de la même famille; c'est le cas de $v$ qui alterne avec $p$ devant -tion (recevoir / réception), de -eux remplacé par -os dans la formation des dérivés en -ité (sinueux / sinuosité), de -qualternant avec -c- dans les dérivés d'adjectifs en -ique 


\section{Elaboration d'un corpus morphophonologique : l'épenthèse consonantique}

(technique / technicien) ou de verbes en -iquer (fabriquer / fabrication), de -eur remplacé par -or devant le suffixe -iser (faveur / favoriser), de -ettre terminaison verbale qui passe à -iss devant -ion (permettre / permission), des adjectifs en -ible et -able qui reçoivent une voyelle supplémentaire lorsqu'ils servent de base à un dérivé (sensible / sensibilité, calculable / calculabilité), etc. La plupart de ces modifications de radical sont implémentables dans l'ordinateur: il suffit de remplacer -ption- par -vtion- (57 termes concernés), -osité- par -euxité- (53 termes), -ique- par -ice (4901 termes), -oris- par -euris- (177 termes), -ission par -ettrion (25 termes) ${ }^{2}$. Certaines ne peuvent faire l'objet d'un traitement automatique sans dommage pour la base de données, comme le remplacement de -er-par -ar- de manière à apparenter mer à maritime: l'alternance est contextuellement justifiable par la présence vs. l'absence d'un morphème suffixal, mais cette donnée n'est pas implémentable dans l'ordinateur.

De même, certaines variations plus ponctuelles ne peuvent faire l'objet d'un remplacement systématique. Ainsi le -t final de court, fort et effort passant à -c- dans accourcir, forcir et efforcer ne subit-il pas la même modification en ce qui concerne désert, import et confort, qui permettent de former respectivement déserter, importer et conforter; un $-x$ final alterne avec -ss- dans certains dérivés (roux / roussir), avec -cpour d'autres (doux / douceur), ou encore avec -s (époux / épouser); un - $d$ - devant le morphème infinitif -er peut être remplacé lors de la dérivation par -s- (corroder / corrosion) ou -ss- (accéder / accession); dans le même contexte, -t- est parfois maintenu (éditer / édition), parfois remplacé par -ss(discuter / discussion), etc. Dans tous ces cas, un traitement automatique de remplacement d'une ou de plusieurs lettres ne donne que peu de résultats pour beaucoup d'erreurs, c'est pourquoi il doit être rejeté. Il faut donc tenir compte, dans les résultats finaux de la recherche «automatique " de termes épenthésés, de ces variations qui produisent autant d'erreurs.

2. Le format du présent article ne permet pas de détailler ces modifications et leur impact dans l'ensemble du corpus. Cf. Pagliano (2003 : 355-361). 


\section{PAGLIANO}

Outre l'allomorphie radicale, qui peut en grande partie être traitée de façon satisfaisante par l'ordinateur, d'autres obstacles à un traitement automatique se dressent. Ainsi les allomorphies suffixales, cruciales puisqu'elles portent sur la présence vs. l'absence d'un son précisément à la frontière avec le morphème radical. Par exemple, le morphème /sj õ/ peut se trouver, en synchronie, sous les formes -tion (distribu-tion), -ation (admir-ation), -ition (impos-ition) et -ion (édit-ion). La confusion est donc possible entre une consonne allomorphique et une consonne épenthétique. C'est pourquoi l'allomorphie suffixale ne peut faire l'objet d'un traitement purement formel comme l'a été l'allomorphie radicale. Elle doit au contraire recevoir une analyse linguistique, qui sera proposée à l'occasion de la deuxième étape de filtre de la base de données.

Une troisième difficulté quant à l'extraction automatique de termes tient aux homographies de radicaux: l'ordinateur identifie comme étant de la même famille communisme et communiquer par exemple, sur la base formelle identique communi- ; lorsque l'homographie est totale, comme dans le cas de briqueterie1 et briqueterie2, l'ordinateur n'a aucun moyen de déceler une épenthèse. Or l'un des termes vient de briquette, mais l'autre est formé sur brique à l'aide du suffixe -erie avec intercalation d'un -t-. Outre le fait qu'il n'associe pas le dérivé avec la base correcte, le traitement automatique appauvrit le corpus d'une unité lexicale épenthésée.

Quand bien même une préparation soigneuse du corpus permettrait de pallier les inconvénients dus aux phénomènes d'allomorphie et d'homographie, se dresse une difficulté supplémentaire : lorsque plusieurs unités lexicales d'une même famille comportent la même consonne épenthétique, la méthode de découpage conduit l'ordinateur à attribuer au radical la consonne en question, au lieu de la considérer comme épenthétique. C'est ainsi qu'il n'identifiera pas le -t- de bijoutier et bijouterie comme une épenthèse.

De même, une consonne épenthétique identique devant un même suffixe conduira le programme à interpréter cette consonne comme faisant partie du suffixe, et non comme une 


\section{Elaboration d'un corpus morphophonologique : l'épenthèse consonantique}

épenthèse. De cette manière, le [t] présent devant le suffixe infinitif dans abriter et biseauter par exemple, bien que correctement identifié comme n'appartenant pas aux bases respectives abri et biseau, sera considéré comme la première lettre du suffixe -ter et non comme une épenthèse.

Il reste enfin à signaler le problème causé par les artefacts graphiques. Dans certains mots en effet, les consonnes finales n'ont pas de réalité étymologique, ce qui conduit légitimement à douter de leur existence lexicale. C'est le cas par exemple du -t de abricot, dont l'étymologie indique qu'il n'est pas présent dans le mot issu de l'arabe al barkouq. De ce fait, le -t- présent dans les dérivés abricoter ou abricotin est vraisemblablement épenthétique, ce que le programme de recherche des épenthèses, parce qu'il est basé sur la graphie, ne permettra pas de mettre en évidence.

Toutes ces difficultés sont néfastes à un traitement automatique satisfaisant du corpus. S'il est vrai que les alternances de radicaux peuvent être traitées en partie, il n'en est pas de même en ce qui concerne les alternances de suffixes et les homographes ; les radicaux de même famille comportant la même épenthèse, tout comme un suffixe donné comportant plusieurs fois la même consonne épenthétique, ne sont pas non plus traitables par la machine, qui n'aura aucun moyen de reconnaître d'éventuelles épenthèses ; enfin, les erreurs dues à la graphie sont difficilement évaluables et quantifiables.

Tous ces motifs concourent au fait que l'analyse automatique, malgré son apparence plutôt prometteuse, produit des résultats médiocres. Ce traitement informatique ne fournit en effet que les épenthèses uniques avec un suffixe donné, pourvu que le radical soit le même dans les deux termes comparés. En ajoutant, après ce travail mécanique, tous les termes de mêmes familles que ceux extraits automatiquement présents dans le TLF et dans le Robert historique, par exemple bleuter lié à bleusaille, on obtient au total 21 dérivés.

La liste de 64.296 termes a été à nouveau utilisée, mais en axant la recherche sur trois suffixes qui se sont révélés, au fur et à mesure de l'avancée de la base de données, parmi les 


\section{PAGLIANO}

plus fréquemment associés à une épenthèse consonantique ${ }^{3}$ : -ier (1238 termes, e.g. bijoutier), -age (2293 termes, e.g. agiotage) et -erie (470 termes, e.g. bijouterie). Trois cent cinquante termes ont été extraits manuellement de ces listes augmentées des mots de même famille: 106 en -er (e.g. queuter), 70 en -ier (e.g. grainetier), 62 en -age (dépiautage), 48 en -erie (e.g. briqueterie), et 64 formés à l'aide d'autres suffixes.

La mise à disposition du Trésor de la Langue Française (TLFi) sur Internet depuis 2001 a permis l'interrogation de cette base de données à partir d'expressions liées à la notion d'épenthèse, telles que "de liaison ", " consonne de », " addition de ", " épenthétique », " parasite ", " transitoire », " graphique », etc., expressions figurant dans le TLFi pour référer à l'épenthèse. Un tri manuel des termes relevés a par la suite été effectué, ce qui a augmenté la base de 48 termes (e.g. brelander, gueuleton).

\subsection{Sources écrites explorées manuellement}

Les apports suivants à la base de données ont été obtenus uniquement au moyen d'une recherche manuelle. Ont ainsi été dépouillés deux dictionnaires d'argot : le Dictionnaire de l'argot français et de ses origines de Colin et al. (1990 [2001]), comportant 7000 termes recensés depuis la fin du XVIII $^{\text {ème }}$ siècle jusqu'à nos jours, et le Dictionnaire du français argotique et populaire de Caradec (2000) proposant 4700 termes. Pour chaque terme listé dans ces dictionnaires, une vérification du radical et du suffixe à l'aide de l'étymologie s'avère nécessaire, de façon à ne pas confondre une épenthèse consonantique éventuelle avec une consonne sous-jacente. Des termes comme papelard, formé à l'aide du suffixe -el et non de la base papier, ou enquiquiner, dérivé avec le suffixe -iner, ont de cette manière été écartés. Soixante-quatre mots se sont ainsi ajoutés à la base de données (e.g. cuterie, sidateux).

3. Le suffixe -er s'est avéré le plus fréquemment attesté précédé d'une épenthèse consonantique, mais il concerne 9992 termes sur 64.296. Le tri de ces termes étant manuel, il ne se justifierait que par une rentabilité intéressante de cette méthode d'extraction, qui ne s'est pas avérée en ce qui concerne les trois suffixes « tests ». 


\section{Elaboration d'un corpus morphophonologique : l'épenthèse consonantique}

Les articles, linguistiques ou non, ont également été sollicités. Il ne s'agit plus d'un dépouillement exhaustif mais d'un relevé aléatoire, en fonction des lectures personnelles, qui a offert 94 termes supplémentaires au corpus (e.g. bénitier, piapiatage).

Enfin, deux recueils de néologismes ont été sollicités : les Néologismes du français contemporain, issus de romans populaires, d'articles de presse et de travaux lexicologiques et lexicographiques, rassemblés par George (2000) entre 1971 et 1991, et l'Insolite, Dictionnaire des mots sauvages, recueillis dans les oeuvres de XIX ${ }^{\text {ème }}$ et $X^{\text {ème }}$ siècles par Rheims (1989). La formation des termes retenus manuellement a été contrôlée au moyen de différents dictionnaires tels que le Dictionnaire des anglicismes (Rey-Debove \& Gagnon 1982), le Dictionnaire de l'argot français et de ses origines (Colin, Mével \& Leclère 1990), le Dictionnaire du français non-conventionnel (Cellard \& Rey 1980) ou le Dictionnaire des mots contemporains (Gilbert 1980), ainsi que dans des dictionnaires plus normatifs tels que le TLF ou le Dictionnaire de la langue française (Littré 1863-1872, suppléments de 1877). Quatre-vingt-six termes ont ainsi complété la base de données (e.g. bocheton, pioutement).

Au total, ce sont 663 termes issus de sources écrites qui ont été rassemblés dans la base de données. Cependant, il était également nécessaire d'exploiter les données orales pour rendre compte au mieux du phénomène d'épenthèse consonantique en synchronie.

\subsection{Place des données orales}

Cent quatre-vingt-seize termes ont été recueillis dans les médias (radio, télévision) ou lors de conversations privées dont 55 en -er, 15 en -ier, 14 en -age, 13 en -eur et 10 en -erie.

Les termes issus de sources orales représentent donc un peu moins d'un quart de la base de données initiale, qui totalise 859 dérivés. Le tableau suivant récapitule et illustre l'apport de chaque source à la base, par ordre décroissant : 


\section{PAGLIANO}

\begin{tabular}{|c|c|c|}
\hline $\begin{array}{l}\text { Type de } \\
\text { source }\end{array}$ & $\begin{array}{c}\text { Nombre } \\
\text { de } \\
\text { termes }\end{array}$ & Termes \\
\hline $\begin{array}{l}\text { Recherche } \\
\text { par suffixe }\end{array}$ & 350 & $\begin{array}{l}\text { aborder ; abricoté ; abricotier ; abricotine ; acclimater ; } \\
\text { accourcir ; aciérage ; afféterie ; affûter ; agiotage ; agioter ; } \\
\text { agioteur ; aiguilletier ; ajouter ; alfatier ; al(l)eutier ; } \\
\text { amadouvier ; arbalétrier ; archeterie ; archetier ; etc. }\end{array}$ \\
\hline $\begin{array}{l}\text { Termes } \\
\text { recueillis à } \\
\text { l'oral }\end{array}$ & 196 & $\begin{array}{l}\text { (dé)marabouter ; aboutir ; aléatoire ; amalfitain ; } \\
\text { amertume ; aquanaute ; bambouseraie ; barbinette ; } \\
\text { bazardage ; bazardement ; bazarder ; bazardeur ; } \\
\text { becqueter ; bijoutaille ; bijouté ; bijouter ; bijouterie ; } \\
\text { bijoutier ; etc. }\end{array}$ \\
\hline $\begin{array}{l}\text { Articles } \\
\text { divers }\end{array}$ & 94 & $\begin{array}{l}\text { abritement ; abriter ; absolutisme ; absolutiste ; aileron ; } \\
\text { amiganesque ; aspergeraie ; bazardier ; beausir ; bénitier ; } \\
\text { blaireauter ; boucautière ; bûcheron ; butorderie ; } \\
\text { cacatesque ; cacatoire ; cachimantier ; etc. }\end{array}$ \\
\hline $\begin{array}{l}\text { Dictionnaires } \\
\text { d'agot }\end{array}$ & 64 & $\begin{array}{l}\text { auverpin ; bagouler ; berlurer ; biffeton ; caf(e)ter ; } \\
\text { cafemar ; cafemon ; cafeteur ; cafeteux ; cafeton ; camtar ; } \\
\text { cinémateux ; colibar ; colibri ; combientième ; conceté ; } \\
\text { cranter ; crapahuter ; crapoteux ; crobard ; etc. }\end{array}$ \\
\hline $\begin{array}{l}\text { Dictionnaire } \\
\text { des mots } \\
\text { sauvages }\end{array}$ & 61 & $\begin{array}{l}\text { abrutiliser ; adultiner ; bedeaudaille ; blaireauteur ; } \\
\text { bleuison ; botocudo ; bouquetin ; bousarderie ; } \\
\text { centaurestique ; chouteur ; crachaté ; déchétique ; } \\
\text { doublezon ; drapeauter ; drapeautique ; égolâtre ; } \\
\text { emperlizer ; etc. }\end{array}$ \\
\hline $\begin{array}{l}\text { Expressions } \\
\text { clés dans le } \\
\text { TLFi }\end{array}$ & 48 & $\begin{array}{l}\text { accoutrer ; arroutain ; atlastique ; bamboutage ; baroufle ; } \\
\text { bourreauder ; brelander ; brelandier ; broutain ; } \\
\text { calembourdier ; calendrier ; calfeutrage ; calfeutrement ; } \\
\text { calfeutrer ; cambrésien ; cambrésine ; chichiter ; etc. }\end{array}$ \\
\hline $\begin{array}{l}\text { Néologismes } \\
\text { du français }\end{array}$ & 25 & $\begin{array}{l}\text { alibababesque ; biberondasse ; biscotonneux ; bochetée ; } \\
\text { bocheton ; bretonnitude ; calorisation ; caloriser ; } \\
\text { cambouité ; cuirture ; émerpiner ; glavioter ; glavioteur ; } \\
\text { hobereautaille ; méliméloter (se) ; pageoterie ; etc. }\end{array}$ \\
\hline $\begin{array}{l}\text { Liste de mots } \\
\text { informatisée }\end{array}$ & 21 & $\begin{array}{l}\text { banlieusard ; bleusaille ; bleuter ; bleuterie ; chevaucher ; } \\
\text { chimiatrie ; diablotin ; dortoir ; harnacher ; joufflu ; } \\
\text { noircir ; portraicturien ; roitelet ; traquenard ; verdâtre ; } \\
\text { verdelet ; verdeur ; verdier ; verdir ; verdoyer ; verdure }\end{array}$ \\
\hline Total & 859 & \\
\hline
\end{tabular}

\subsection{Bilan : 859 dérivés, 89 terminaisons différentes}

A ce stade de la constitution du corpus, pas moins de 89 finales différentes sont attestées, dont seize seulement concernent au moins dix dérivés chacune et quarante-trois ne figurent que dans un seul dérivé. 
Elaboration d'un corpus morphophonologique : l'épenthèse consonantique

\begin{tabular}{|c|c|c|}
\hline Finales & $\begin{array}{l}\text { Nombre } \\
\text { termes / } \\
\text { finale }\end{array}$ & Illustrations \\
\hline -er & 226 & abriter ; aborder ; boiser ; adultiner... \\
\hline -ier & 99 & abricotier ; bazardier ; jambosier... \\
\hline -age & 85 & agiotage ; badaudage ; masselottage... \\
\hline -erie & 75 & afféterie; badauderie ; baguenauderie... \\
\hline -eur & 32 & agioteur ; argoteur ; bardeur ; bazardeur... \\
\hline -on & 26 & biffeton ; aileron ; bûcheron ; écoinçon... \\
\hline -esque & 26 & cacatesque ; ceaucesculesque... \\
\hline -é & 25 & abricoté ; bijouté ; barreaudé ; enképissé... \\
\hline -ique & 20 & argotique ; atlastique ; bureautique... \\
\hline -eux & 18 & cafeteux ; cafardeux ; cauchemardeux... \\
\hline -ement & 14 & empiètement ; ensorcellement... \\
\hline -isme & 11 & absolutisme ; argotisme ; favoritisme... \\
\hline -ure & 10 & cuirture ; fermeture ; fourniture... \\
\hline -iser & 10 & climatiser ; eczématiser $\left(s^{\prime}\right)$; médiatiser $1 . .$. \\
\hline -ière & 10 & boucautière ; cacaotière ; coquetière... \\
\hline -ard & 10 & banlieusard ; bondieusard ;vicelard ... \\
\hline -in & 9 & bouquetin ; diablotin ; fagotin ;tableautin... \\
\hline -ir, -ain, -? & 7 & durcir / arroutain ; broutain / vrille... \\
\hline -eau, -aille, -ois & 6 & lionceau / piétaille / souriquois ;graylois \\
\hline -oire, -ine, -ien, -ie & 5 & aléatoire / myrosine / lunaisien / tabagie... \\
\hline -ation, -ais & 4 & calorisation / togolais ; javanais... \\
\hline $\begin{array}{l}\text {-té, -iste, -ette, -et, } \\
\text {-elet, -âtre, -ar, -ant, } \\
\text {-al, -aire }\end{array}$ & 3 & $\begin{array}{l}\text { conceté / argotiste / barbinette/ coqueret / } \\
\text { verdelet / hugolâtre / cafemar / loftstorysant } \\
\text { / horizontal / sursitaire ; tissulaire... }\end{array}$ \\
\hline $\begin{array}{l}\text {-ude, -ot, -go, -fier, } \\
\text {-ée, -aute, -able }\end{array}$ & 2 & $\begin{array}{l}\text { dégourditude / gosselot / icigo / cocufier / } \\
\text { bochetée / aquanaute / catégorisable }\end{array}$ \\
\hline $\begin{array}{l}\text {-urien, -ume, -uche, } \\
\text {-u, -ri, -re, -oyer, } \\
\text {-ourde, -otter, -otte, } \\
\text {-oque, -ologue, -oir, } \\
\text {-o, -lu, -ium, -ite, -is, } \\
\text {-ion, -ingue, -iner, } \\
\text {-ille, -ifier, -if, -ième, } \\
\text {-iat, -euse, -eter, } \\
\text {-esse, -eraie, -elle, } \\
\text {-eler, -éen, -dé, } \\
\text {-caille, -axie, -asser, } \\
\text {-asse, -ance, -an, -aie, } \\
\text {-(o)logie, -(ill)age }\end{array}$ & 1 & $\begin{array}{l}\text { portraicturien / amertume / coqueluche / } \\
\text { chevelu / colibri / coudre / verdoyer / } \\
\text { coquelourde / picoter / masselotte / } \\
\text { chinetoque / sidénologue / pourrissoir / } \\
\text { botocudo / joufflu / mycélium / halloysite / } \\
\text { cailloutis / taxation / louftingue / piétiner / } \\
\text { brindille / salifier / taxatif / combientième / } \\
\text { marloupiat / émeriseuse / piqueter / } \\
\text { têtutesse / bambouseraie / coquerelle / } \\
\text { ensorceler / sabatéen / jourdé / icicaille / } \\
\text { zootaxie / avocasser / biberondasse / } \\
\text { cuistance / partisan / aspergeraie / } \\
\text { climatologie / enfantillage }\end{array}$ \\
\hline 89 & 859 & \\
\hline
\end{tabular}




\section{PAGLIANO}

Cette hétérogénéité de la base de données montre la nécessité de filtrer ces dérivés : il faut vérifier la réalité de l'épenthèse supposée de façon à s'assurer qu'il ne s'agit pas d'une consonne présente dans le lexique qui ne se manifesterait que sous certaines conditions.

\section{Traitement de la base de données : élaboration du corpus de travail}

La base de données va passer par deux filtres différents. Le premier consistera à l'épurer des termes pour lesquels l'épenthèse n'est pas à l'endroit souhaité et de ceux pour lesquels l'étymologie ou les alternances radicales ou suffixales excluent l'épenthèse. Un second filtre s'intéressera plus particulièrement aux suffixes devant lesquels semble se produire une épenthèse ; il tâchera d'étudier chaque cas en fonction des bases possibles et des signifiés de la base, du suffixe et du terme dérivé.

\subsection{Filtre étymologique: focalisation sur la dérivation suffixale en français}

Les deux premières causes de rejet d'un terme de la base sont extérieures à la dérivation : il s'agit de l'introduction malheureuse d'unités lexicales composées et non dérivées, ou dont l'épenthèse ne se situe pas entre le radical et le suffixe. Six termes se sont avérés relever du phénomène de composition et non de dérivation, ce qui les écarte d'emblée de l'étude: hugolâtre, coquelourde, aquanaute, cosmonaute, zootaxie, égolâtre. Par ailleurs, huit des termes recueillis par la recherche de séquences clés telles que "épenthèse " ou "consonne de transition » dans le TLFi ne contiennent pas l'épenthèse entre radical et suffixe mais ailleurs dans le mot, ce qui les exclut du champ de cette étude: baroufle, cible, coudre, engendrer, filandre, polacre, tringle, vrille.

Un troisième motif d'exclusion de la base est lié à l'étymologie du terme considéré. Le but d'une recherche étymologique dans le cadre de cette étude est double. Il s'agit en premier lieu d'écarter les consonnes qui apparaissent comme une épenthèse en français aujourd'hui, mais qui en fait ont un représentant en latin (voire en grec) comme le [t] d'amertume, 


\section{Elaboration d'un corpus morphophonologique : l'épenthèse consonantique}

qui vient du latin amaritudinem. Le second objectif consiste à écarter les consonnes actuellement sous-jacentes mais qui ont été présentes à un moment donné, dont on peut supposer qu'elles ont laissé une trace en synchronie dans le morphème ; ainsi [z] dans bleusaille, candidat au statut d'épenthèse, est disqualifié du fait que le terme d'où il est issu est dérivé de bleuse, féminin dialectal de bleu. En revanche, les «fausses » épenthèses, c'est-à-dire celles qu'une étymologie populaire ou un peu hâtive indiqueraient comme telles sans qu'elles en soient (clouter < gallo-roman clouster, dérivé de clouet et non de clou), souvent mises en évidence dans les dictionnaires, n'ont pas été écartées. En effet, dans quelle mesure l'interprétation populaire de la formation de ces termes intervient-elle dans l'analogie ? Donc dans la création d'épenthèses?

Trois dictionnaires ont été utilisés pour établir l'étymologie des unités lexicales: le Trésor de la Langue Française (version papier puis informatisée), le Robert, Dictionnaire historique de la langue française (1992 [2000]) et le Dictionnaire étymologique de la langue française de Bloch \& Wartburg (1932 [1968]). Consulter ces dictionnaires permet également de distinguer dans certains cas des homophones formés sur des bases différentes, dont l'un présente une épenthèse intéressante, comme dans le cas de briqueterie détaillé en section 1.3. En revanche, les dictionnaires ne seront pas considérés comme arbitres dans la décision finale relative à une consonne candidate à l'épenthèse dans un terme donné. Prenons, pour définir le propos, le cas du dérivé agiotage. Le TLF le considère comme dérivé du verbe agioter, alors que Bloch \& Wartburg le rattachent directement à agio. Dans un cas il y a épenthèse et le terme est intéressant pour l'analyse, dans l'autre l'épenthèse n'est qu'« empruntée ». Il en est de même pour le terme biseautage : le TLF et Rey \& al. (1992) le lient au verbe biseauter, mais Bloch \& Wartburg (1932) le dérivent à partir du substantif biseau. Que faire si l'on a pris comme critère décisionnel absolu l'avis des dictionnaires étymologiques et qu'il ne s'entendent pas ? Il conviendra plutôt de prendre une décision en fonction de l'étude du suffixe -age et des bases auxquelles il peut s'adjoindre, concernant l'ensemble des dérivés 


\section{PAgLiano}

en -age : sont-ils tous rattachables à un verbe en -er, attesté ou virtuel ? Ce n'est qu'au terme du second filtre, exposé en section 3.2, que l'on pourra statuer sur les termes concernés.

Les raisons liées à l'étymologie permettant de décider de la présence d'une unité lexicale au sein du corpus final, sont au nombre de cinq, dont trois en faveur du rejet des termes concernés. Un premier ensemble de termes sera en effet exclu du fait qu'il ne s'agit pas d'unités lexicales dérivées en français, mais que la consonne potentiellement intéressante appartient en réalité à un terme latin. Vingt-trois termes sont ainsi rejetés, comme calendrier (du bas latin calendarium) ou fermeté (du latin firmitas). Les bases qui comportent une consonne sousjacente à la finale, indiquée par leur étymologie, disqualifient les dérivés associés, soit 229 termes parmi lesquels diablotin (dérivé de diablot) ou verdure (vert est issu du latin viridis ; on trouve d'ailleurs également la forme verde au moyen-âge). Dans deux termes, la consonne potentiellement épenthésée n'est que graphique et ne correspond à aucun élément étymologique ni phonologique: $-c$ - dans portraicturien et $-p$ - dans dompter. Trois dérivés font l'objet d'un croisement de plusieurs étymologies, rendant malcommode l'appréhension de leur formation: coquebin (dérivé de coq ou de coquebert par substitution de suffixe), vivandier (réfection de l'ancien français viandier, d'après le latin vivenda ou sous l'influence de vivres) et déposer (emprunt au latin deponere avec francisation d'après poser); il n'est pas possible, au moins à cette étape de leur traitement, de décider de leur maintien ou de leur rejet dans le corpus. Pour soixante-quatre termes enfin, l'étymologie indique une consonne en finale de base, mais non la même que celle qui est épenthésée dans le dérivé correspondant: empiéter ou miroitier comportent un [t] candidat, au lieu du [d] ou du [r] attendus respectivement à cause de l'étymologie (empiéter est dérivé sur pied, du latin pedem, miroitier est construit à partir de miroir). Ces unités lexicales sont maintenues dans le corpus au terme de ce premier filtrage. 
Elaboration d'un corpus morphophonologique : l'épenthèse consonantique

\begin{tabular}{|c|c|c|c|}
\hline $\begin{array}{l}\text { Détail du rôle de } \\
\text { l'étymologie dans le } \\
\text { premier filtre }\end{array}$ & $\begin{array}{l}\text { Traitement } \\
\text { après 1er } \\
\text { filtre }\end{array}$ & $\begin{array}{l}\text { Nombre } \\
\text { termes } \\
\text { concernés }\end{array}$ & Illustrations \\
\hline $\begin{array}{l}\text { héritage d'un mot déjà } \\
\text { dérivé avec la consonne } \\
\text { en latin }\end{array}$ & rejeté & 31 & $\begin{array}{l}\text { chevaucher ; fermeté ; } \\
\text { hiverner ; noircir ; } \\
\text { aléatoire }\end{array}$ \\
\hline $\begin{array}{l}\text { présence de la consonne } \\
\text { dans l'étymologie de la } \\
\text { base }\end{array}$ & rejeté & 226 & $\begin{array}{l}\text { diablotin ; emperlousé ; } \\
\text { nagualisme ; cordelier }\end{array}$ \\
\hline $\begin{array}{l}\text { consonne uniquement } \\
\text { graphique }\end{array}$ & rejeté & 2 & portraicturien ; dompter \\
\hline $\begin{array}{l}\text { croisement de plusieurs } \\
\text { étymologies }\end{array}$ & indécision & 3 & $\begin{array}{l}\text { coquebin ; vivandier ; } \\
\text { déposer }\end{array}$ \\
\hline \multirow[t]{2}{*}{$\begin{array}{l}\text { l'étymologie présente } \\
\text { une consonne différente } \\
\text { de celle de l'épenthèse }\end{array}$} & maintien & 52 & $\begin{array}{l}\text { abricotier ; jutage ; } \\
\text { reinté ; faisander ; } \\
\text { dieutelet ; taillandier ; } \\
\text { juteux ; tabatière }\end{array}$ \\
\hline & & 314 & \\
\hline
\end{tabular}

L'étymologie des dérivés et des bases a permis de mettre en évidence les consonnes sous-jacentes de 259 termes. Ce n'est pas cependant la seule méthode de mise au grand jour des consonnes sous-jacentes. Celles-ci peuvent en effet apparaître à la liaison (un petit [t] enfant) ou au féminin (une grande amie). Les termes pour lesquels il est possible d'accéder au féminin, à savoir les adjectifs qualificatifs et certains substantifs, ont donc bénéficié d'un garde-fou supplémentaire destiné à s'assurer du caractère réellement épenthétique de la consonne suspecte entre radical et suffixe. Le tamis étymologique ayant déjà éliminé la plupart d'entre eux, ne restent ici que les termes pour lesquels le féminin fait apparaître une consonne sans que cette consonne ne soit présente dans l'étymologie du mot. Parmi les 19 termes concernés (favoritisme, butorderie, etc.), six sont formés à partir de bases en -aud, deux de base en - $a t$, trois de bases en -[u] et quatre sont de la famille de gueux. 


\section{PAGLIANO}

\begin{tabular}{|l|l|l|}
\hline Finale de la base & Dérivés concernés & $\begin{array}{l}\text { Nombre de } \\
\text { bases }\end{array}$ \\
\hline -aud & $\begin{array}{l}\text { badaudage ; badauder ; badauderie ; } \\
\text { esquimaudage ; finauderie ; } \\
\text { rustauderie }\end{array}$ & 4 bases \\
\hline -at & rapiater ; rapiaterie & 1 base \\
\hline -ou & chouchouter ; voyouter ; chouteur & 3 bases \\
\hline -eux & $\begin{array}{l}\text { gueusaille ; gueusard ; gueuser ; } \\
\text { gueuserie }\end{array}$ & 1 base \\
\hline autres : -i, -ois, -ord & favoritisme ; matoiserie ; butorderie & 3 bases \\
\hline
\end{tabular}

Finaud et rustaud sont formés à l'aide du suffixe $-\operatorname{aud}(e)$, ce qui indique que [d] dans les dérivés est sous-jacent et non épenthétique. En revanche, badaud étant emprunté au provençal badau et esquimau à une langue amérindienne, on pourrait considérer que [d] est bien épenthétique dans badaud et esquimau. Cependant, le féminin de badaud se fait à l'aide d'un [d], ce qui semble indiquer la réfection du suffixe -aud et disqualifie le terme de la base de données. Ce traitement peut être étendu à esquimau.

L'origine de rapiat n'est pas nette ; puisqu'il n'est pas exclu de rattacher cette base au suffixe -at issu du latin -atus, qui comporte donc vraisemblablement un /t/ lexical, force est d'exclure les dérivés rapiater et rapiaterie du corpus.

Les quatre dérivés de gueux comportent [z] entre la base et le suffixe, qui n'est pourtant pas présent dans l'étymologie du terme: gueux est emprunté au moyen néerlandais guit sous la forme gueu, le - $x$ en fin de base n'étant que graphique. Cependant, les dictionnaires proposent d'expliquer l'apparition du [z] au féminin, et a fortiori dans la dérivation, par une réinterprétation de la terminaison par le suffixe -eux. La présence de la consonne au féminin de l'adjectif exclut de ce fait les quatre dérivés de la base.

Les trois dérivés à partir de bases en -[u] comportent la même consonne au féminin : [t]. La relative importance de cette terminaison est-elle indicatrice d'un phénomène d'épenthèse propre à ce contexte ? Une fois l'ensemble du corpus totalement traité, il sera intéressant d'établir l'existence éventuelle d'une 


\section{Elaboration d'un corpus morphophonologique : l'épenthèse consonantique}

tendance générale à épenthéser une consonne après [u] et si cette consonne est bien [t].

Du fait que l'étymologie n'a pas établi de consonne sous-jacente pour les dix-neuf termes examinés ici (e.g. favoritisme), la consonne qui apparaît au féminin est épenthétique dans le sens où elle a été créée ex nihilo. Il n'est cependant pas possible de déterminer, pour huit cas (e.g. chouchouter) sur les dix-neuf, si c'est le féminin qui a, le premier, reçu l'épenthèse pour ensuite la communiquer à la dérivation, ou l'inverse. C'est pourquoi tous ces termes seront exclus du corpus.

Ce premier filtre prend également en charge les alternances en début de suffixe du dérivé et celles en finale de radical.

Les suffixes concernés sont d'une part les suffixes verbaux -iser, -et $(t) e r$ et -ot $(t) e r$ en relation avec -er, d'autre part certains suffixes argotiques.

Le corpus compte 21 termes se terminant par l'une des séquences -iser, -iseur, -iseuse, -isant ou -isable, parmi lesquels infiniser ou loftstorysant. Merceriser, issu de l'anglais mercerize, est directement rejeté du corpus. Le traitement des vingt unités lexicales exige en revanche une analyse de leur signifié : pour sept d'entre elles en effet, le sens du dérivé correspond à «rendre $\mathrm{X}$ » ou "transformer en $\mathrm{X}$ » (e.g. favoriser), $\mathrm{X}$ représentant la base, ce qui indique une formation à partir du suffixe -iser et non une épenthèse ; c'est pourquoi elles sont rejetés de la base. Le signifié des treize termes restants ne met en revanche pas en évidence leur formation à partir du suffixe -iser, aussi sont-ils maintenus dans le corpus. De même, les 42 unités lexicales en -et(t)er se répartissent en fonction de l'adéquation de leur signifié avec l'idée de diminutif véhiculée par -et, ou de fréquence par -eter : les dix-sept termes qui s'y conforment sont rejetés, dix-sept autres ne peuvent être mis en relation avec les suffixes suscités et sont donc considérés formés à l'aide du suffixe -er et d'une épenthèse ; pour huit enfin il n'est pas possible d'établir avec certitude la base. Enfin, quinze des trente-sept termes en -ot(t)er de la base de données 


\section{PAGLIANO}

sont rejetés puisque leur signifié intègre l'idée de diminutif ou de fréquence, pour dix-sept il n'est pas possible de prendre une décision définitive, seuls sept sont maintenus dans le corpus.

\begin{tabular}{|l|c|l|c|l|}
\hline Suffixe & $\begin{array}{c}\text { Nombre } \\
\text { rejetés / en } \\
\text { balance }\end{array}$ & Termes rejetés / en balance & $\begin{array}{c}\text { Nombre } \\
\text { maintenus }\end{array}$ & Termes maintenus \\
\hline -iser & 8 & $\begin{array}{l}\text { merceriser ; favoriser ; } \\
\text { tatamiser ; infiniser ; } \\
\text { stromboliser ; symétriser ; } \\
\text { symétriseur ; rouliser }\end{array}$ & 13 & $\begin{array}{l}\text { colorisation ; émerisage ; } \\
\text { émeriser ; émeriseuse ; } \\
\text { taniser ; catégorisable ; } \\
\text { catégorisation ; } \\
\text { catégoriser ; } \\
\text { loftstorysant ; } \\
\text { macadamiser ; } \\
\text { trotskisant ; névralgiser ; } \\
\text { phraséologisé }\end{array}$ \\
\hline -et(t)er & 17 / 8 & $\begin{array}{l}\text { archeterie ; archetier ; } \\
\text { bouvetage ; grainetier2 ; } \\
\text { guichetier ; marqueterie ; } \\
\text { paquetage ; paqueter ; } \\
\text { paqueteur ; paquetier ; } \\
\text { parcqueter ; robinetterie ; } \\
\text { louveteau / } \\
\text { bonneter ; bonnetier ; } \\
\text { bufettier ; courtage ; } \\
\text { courtier ; épincetage ; } \\
\text { épousseter ; gobetage }\end{array}$ & 17 & $\begin{array}{l}\text { buffleterie ; chainetier ; } \\
\text { coquetier1 ; écoquetage ; } \\
\text { écoqueter ; feuilleter ; } \\
\text { giletier ; giletière ; } \\
\text { grainetier1 ; graineterie ; } \\
\text { museletage ; pelleter ; } \\
\text { pelleterie ; pelletier ; } \\
\text { clouter ; clouterie : } \\
\text { déchétique }\end{array}$ \\
\hline -ot(t)er & $\begin{array}{l}\text { ilotage ; îlotier ; minotier ; } \\
\text { tricoter ; verroterie ; } \\
\text { chevroter ; pageoterie ; } \\
\text { picoter ; cachotter ; } \\
\text { cachotterie ; cachottier / } \\
\text { argotage ; argoter ; } \\
\text { argoteur ; argotier ; } \\
\text { argotique ; argotisme ; } \\
\text { argotiste ; bimbeloterie ; } \\
\text { cagoterie ; crabotage ; } \\
\text { ergoter ; fagoter ; fagotier ; } \\
\text { fagotin ; palotage ; rabioter ; } \\
\text { déposer }\end{array}$ & 7 & $\begin{array}{l}\text { canotier ; lingotier ; } \\
\text { fayotage ; fayoter ; } \\
\text { fayoteur ; glavioter ; } \\
\text { glavioteur }\end{array}$ \\
\hline
\end{tabular}

Une place à part doit être réservée aux suffixes argotiques, dans le sens où ce n'est pas le signifié qui donne une 


\section{Elaboration d'un corpus morphophonologique : l'épenthèse consonantique}

indication de l'identité du suffixe, mais le registre de langue. Ces suffixes sont d'ailleurs identifiés comme tels par Colin, Mével \& Leclère (1990). Certains dérivés ont donc été écartés, parce que dérivés à l'aide d'un suffixe présentant une consonne à l'initiale ; ainsi 27 termes, parmi lesquels ceux formés à partir des suffixes -ton (biffeton, mecton, etc.), -go (icigo, labago) et -tance (cuistance) par exemple, ont-ils été écartés, sur les 29 dérivés argotiques que comportait la base de données. Vicelard et faflard, parce que le suffixe -lard n'est pas explicitement relevé dans les dictionnaires au contraire du suffixe -ard, sont en effet maintenus.

Vingt-sept termes enfin sont éliminés ou mis de côté dès ce premier filtre. Pour huit d'entre eux, les suffixes avaient été mal identifiés lors de la création de la base de données initiale, pour des raisons orthographiques (bochetée), de cumul de suffixes (chimie +- -atre $+-i e$ ), de manque d'analyse du signifié (statufier, cocufier sont formés à l'aide du suffixe -(i)fier ; royauté, avocasser) ou de manque d'information sur le suffixe final de la base (pensementer, pensementerie). Des dixneuf termes restants, on ne peut avec certitude décider le maintien ou le rejet; six sont de la famille de coq, la base pouvant être coquel (coquerelle, coquelet, coqueret, coqueleur, coqueleux, coqueliner) ; quatre présentent une variante suffixale possible mais non répertoriée dans les dictionnaires (iglouchon, bretonnitude, pourriture, fourniture) ; neuf termes sont formés à partir des suffixes -ette et -on dont les variantes -elette (sourcelette, tartelette) et -eron (aileron; bûcheron; mancheron1 ; mancheron2 ; tâcheron ; moucheron ; vigneron) sont répertoriées et attestées plusieurs fois, mais sans qu'elles correspondent à un signifié particulier. Pourquoi tarte se voit adjoint la variante -elette et non -ette, le signifié étant dans les deux cas «petite»? Pourquoi une petite mouche est-elle indiquée par le signifiant moucheron et non mouchon?

En ce qui concerne le traitement plus particulier des suffixes, ce sont 151 unités lexicales qui ont été étudiées dont seules 38 ont été maintenues. 


\section{PAGLIANO}

La dernière étape de ce premier filtre consiste à étudier les alternances radicales. Deux cas se distinguent: les bases incertaines d'une part, la présence de certains suffixes en fin de base d'autre part.

La base de données comporte 61 dérivés dont la forme de la base est incertaine. Six d'entre eux seulement sont maintenus dans le corpus final, ceux pour lesquels parmi les bases proposées, au moins une est actuelle, c'est-à-dire réanalysable en synchronie comme étant dérivée dans le français contemporain: bleusaille, chalutage, chaluter, chalutier, dinanderie, dinandier. L'exemple phare est bleusaille : en synchronie, ce terme est réanalysé comme étant formé sur bleu et non sur le féminin dialectal bleuse. Ce n'est que l'examen du suffixe au terme de l'analyse qui permettra éventuellement d'écarter par la suite ce dérivé. Trente-sept termes restent frappés du sceau de l'indécision, soit parce que plusieurs bases sont possibles (ridelage, englumer), soit que la base reste obscure (sidénologue, manchereau). Là encore, seuls les filtres suivants permettront de reclasser une partie d'entre eux. Si toutes les bases proposées par les dictionnaires comportent la consonne que l'on trouve dans le dérivé à leur finale, les dérivés sont rejetés car on ne peut exclure que la consonne soit présente à la finale de la base dans le lexique ; dix-huit termes sont ainsi exclus du corpus : verdâtre, verdelet, verdeur, verdier, verdir, verdoyer, verdure; calfeutrage, calfeutrement, calfeutrer; conchyoline ; partisan ; bouverie ; forestier ; ponceau ; chevelure ; ceilleton; soudaineté.

Parmi les terminaisons possibles d'une base, quatre suffixes se distinguent. Les suffixes -aud et -at ont déjà été évoqués lors de l'étude du féminin des adjectifs. Cependant, lorsque la base n'est pas attestée au féminin (minaud), seule l'étymologie permet de statuer sur le rejet du dérivé concerné : le suffixe -aud est issu du francique -wald, de walden " gouverner », le suffixe comporte donc un /d/ lexical. Crachaté est formé à partir du suffixe -at provenant du latin -atus, taxateur et taxatif présentent un élément -at, peut-être dû à un allongement thématique, et seront donc évalués par le deuxième filtre. Gravatier et goujaterie, formés sur gravois et d'un terme 


\section{Elaboration d'un corpus morphophonologique : l'épenthèse consonantique}

provençal en [a], sont maintenus. Le suffixe -ard est issu de l'adjectif allemand hart "dur», c'est pourquoi il semble légitime de supposer que le suffixe français comporte un /d/ lexical final, aussi foulardage, bousarderie, musarderie et jobarder sont-ils exclus du corpus. Il en va de même pour roulis, dont le suffixe -is provient d'une forme -ëiz en ancien français, du latin -aticiu.

Résumons-nous : au terme du premier filtre, sur 531 dérivés examinés, seuls 98 sont maintenus dans la base de données, soit moins d'un cinquième. Des 859 unités lexicales de la base de départ, 422 sont encore présents, soit un peu moins de la moitié. On notera que pour une même famille lexicale, différents traitements des dérivés sont observés. Considérons par exemple la famille de coq, qui comporte 14 dérivés dans la base initiale. Un terme est éliminé car il est composé et non dérivé ; pour huit dérivés, il n'est pas possible de prendre de décision car la base est incertaine pour sept d'entre eux et plusieurs bases sont possibles pour le huitième ; cinq enfin sont maintenus, soit parce qu'ils n'ont pas donné lieu à une évaluation, soit parce que l'évaluation a conclu au caractère épenthétique de la consonne candidate.

\begin{tabular}{|c|c|c|c|c|}
\hline & $\begin{array}{l}\text { Nombre } \\
\text { de } \\
\text { dérivés }\end{array}$ & Justification & $\begin{array}{l}\text { Nombre } \\
\text { de } \\
\text { dérivés }\end{array}$ & Termes \\
\hline Eliminés & 1 & Composé & 1 & coquelourde \\
\hline \multirow[t]{2}{*}{ Indécidés } & \multirow[t]{2}{*}{8} & Base incertaine & 7 & $\begin{array}{l}\text { coqueleux; coqueliner ; } \\
\text { coquelet ; coqueleur ; } \\
\text { coqueluche ; coquerelle; } \\
\text { coqueret }\end{array}$ \\
\hline & & $\begin{array}{l}\text { Plusieurs bases } \\
\text { possibles }\end{array}$ & 1 & coquebin \\
\hline \multirow{3}{*}{$\begin{array}{l}\text { Maintenu } \\
\mathrm{s}\end{array}$} & \multirow[t]{2}{*}{5} & Finale en -eter & 3 & $\begin{array}{l}\text { coquetier } 2 \text {; coquetière ; } \\
\text { coquetier } 1\end{array}$ \\
\hline & & Non évalués & 2 & écoquetage ; écoqueter \\
\hline & 14 & & & \\
\hline
\end{tabular}

Ce filtre à orientation étymologique a été complété par un second plus directement axé sur la morphologie puisqu'il 


\section{PAGLiAnO}

porte sur l'examen de chacun des 50 suffixes identifiés à l'issue du premier filtre, que le tableau ci-dessous récapitule :

\begin{tabular}{|c|c|c|}
\hline Suffixe & $\begin{array}{l}\text { Nombre } \\
\text { termes par } \\
\text { suffixe }\end{array}$ & Exemples de termes \\
\hline -er & 108 & abriter ; bazarder ; caloriser ; adultiner... \\
\hline -ier & 49 & $\begin{array}{l}\text { abricotier ; al(l)eutier ; bazardier ; } \\
\text { boyaudier... }\end{array}$ \\
\hline -age & 36 & dépiautage ; barreaudage ; émerisage... \\
\hline -erie & 32 & $\begin{array}{l}\text { bijouterie ; bleuterie; boyauderie; } \\
\text { dinanderie... }\end{array}$ \\
\hline -esque & 24 & $\begin{array}{l}\text { cacatesque ; ceaucesculesque ; } \\
\text { amiganesque... }\end{array}$ \\
\hline -eur & 21 & $\begin{array}{l}\text { agioteur ; biseauteur ; blaireauteur ; } \\
\text { bazardeur... }\end{array}$ \\
\hline -é, -eux, -ique & 13 & $\begin{array}{l}\text { abricoté / caillouteux / atlastique ; } \\
\text { bureautique... }\end{array}$ \\
\hline -ement & 11 & $\begin{array}{l}\text { abritement ; glougloutement ; } \\
\text { bazardement... }\end{array}$ \\
\hline -ière & 8 & $\begin{array}{l}\text { cacaotière ; cafetière ; coquetière ; } \\
\text { giletière... }\end{array}$ \\
\hline -ain, -iser & 6 & $\begin{array}{l}\text { amalfitain ;valéricain / médiatiser ; } \\
\text { radiotiser... }\end{array}$ \\
\hline -ure, -ois, -isme & 5 & $\begin{array}{l}\text { cuirture / souriquois ; graylois / } \\
\text { gagatisme... }\end{array}$ \\
\hline $\begin{array}{l}\text {-aille, -ais, -ard, } \\
\text {-ation, -ien, -ine }\end{array}$ & 4 & $\begin{array}{l}\text { bijoutaille / congolais / queutard / } \\
\text { calorisation / onusienne / indigotine... }\end{array}$ \\
\hline -aire, -ant, -in, -té & 3 & $\begin{array}{l}\text { sursitaire / glougloutant / piétin / } \\
\text { faucuseté... }\end{array}$ \\
\hline -elet, -ir, -ot & 2 & dieutelet / glougloutir / gosselot ; queusot... \\
\hline $\begin{array}{l}\text {-able, -al, -asse, } \\
\text {-eau, -ée, -éen, eraie, } \\
\text {-esse, -ette, -euse, } \\
\text {-ie, -ième, -if, -ille, } \\
\text {-iner, -is, -ite, -oire, } \\
\text {-on, -otte, -ude }\end{array}$ & 1 & $\begin{array}{l}\text { catégorisable / soucutal / biberondasse / } \\
\text { faiseandeau / yeutée / sabatéen / } \\
\text { bambouseraie / têtutesse / barbinette / } \\
\text { émeriseuse / papousie / combientième / } \\
\text { taxatif / brindille / piétiner / cailloutis / } \\
\text { halloysite / cacatoire / piéton / masselotte / } \\
\text { dégourditude }\end{array}$ \\
\hline 50 suffixes & 422 & \\
\hline
\end{tabular}


Elaboration d'un corpus morphophonologique : l'épenthèse consonantique

\subsection{Filtre morphologique: de la place et de la forme des bases et des suffixes}

Il s'agit, dans cette deuxième étape d'épuration de la base de données, d'établir s'il s'agit bien du suffixe supposé, en fonction de deux critères : le type de base auquel le suffixe est réputé s'adjoindre, et surtout les signifiés, celui du suffixe comme celui du dérivé considéré. L'étude des suffixes s'appuie notamment sur les travaux lexicologiques de Corbin (1987) et Dubois \& Dubois-Charlier (1999). La philosophie de la procédure est la suivante : s'il existe ne serait-ce qu'une analyse mettant en doute la formation d'un dérivé à l'aide d'une épenthèse consonantique, parce que plusieurs bases sont possibles ou que certains allomorphes suffixaux comportent la même consonne, le dérivé est exclu de l'ensemble des termes maintenus. Considérons par exemple le terme cailloutis ; s'il est formé à partir du verbe caillouter, il ne comporte pas d'élément épenthétique, mais s'il est formé sur le substantif caillou il contient l'élément "parasite" [t]. Seule la base verbale est attestée avec ce suffixe (roulis sur rouler, gribouillis sur gribouiller ; cf. Corbin 1987 : 683, Dubois \& Dubois-Charlier 1999: 38-40, Apothéloz 2002: 76), ce qui indique que cailloutis est construit à partir du verbe et non du substantif ; il ne contient donc pas de consonne épenthétique et à ce titre est éliminé du corpus.

Cette position se traduit par une réduction de la base de données de 422 à 259 dérivés, dont la fiabilité en regard de l'analyse envisagée est renforcée. Une place particulière a été accordée à l'étude des terminaisons en -ar et en -iser, en relation avec les suffixes -ard et -iser : l'homophonie des terminaisons avec les suffixes, bien que le sens atteste de l'absence de ces derniers, a pu jouer un rôle dans l'épenthèse de [d] et [z] respectivement après -ar (caviarder) et entre une base en -[i] et un suffixe infinitif en -er (trotskyser). Le détail pour chaque terme évalué ne peut être fourni ici faute de place, on le trouvera dans Pagliano (2003 : 453-526). Par ailleurs, l'examen des signifiés des dérivés en -age, -aire, -ant, -ation, -eur et -ure, en regard des signifiés de ces suffixes, a conduit à poser 


\section{PAgLiano}

l'existence de 13 termes virtuels ( ${ }^{\circ}$ escargoter ${ }^{4}$, ${ }^{\circ}$ siloter, ${ }^{\circ}$ yeuter, etc.) ; ainsi loftstorysant est-il exclu du corpus puisque son sens est paraphrasable par " qui loftstoryse ", ce qui correspond au suffixe -ant "qui $\mathrm{V}$ » et indique donc la formation à partir du verbe virtuel ${ }^{\circ}$ loftstoryser. Le tableau suivant fournit le traitement global des 435 dérivés (422 à l'issue du premier filtre augmentés des 13 virtuels) en fonction des suffixes (-et et -ette ont été regroupés, de même que -ien et -éen, -eux et -euse, -ot et -otte) :

\begin{tabular}{|c|c|c|c|c|}
\hline Suffixe & Illustrations & $\begin{array}{c}\text { Termes } \\
\text { en } \\
\text { balance }\end{array}$ & $\begin{array}{l}\text { Termes } \\
\text { rejetés }\end{array}$ & $\begin{array}{c}\text { Termes } \\
\text { maintenus }\end{array}$ \\
\hline -eau & faisandeau & & & 1 \\
\hline -(el)et(te) & dieutelet, roitelet, barbinette & & & 3 \\
\hline -in & gosselin, piétin, tableautin & & & 3 \\
\hline -ain & $\begin{array}{l}\text { amalfitain, broutain, samaritain, } \\
\text { valéricain }\end{array}$ & & & 6 \\
\hline -ais & $\begin{array}{l}\text { congolais, togolais, javanais, } \\
\text { vinçanais }\end{array}$ & & & 4 \\
\hline -ois & $\begin{array}{l}\text { spadois, graylois, souriquois, } \\
\text { pouillyzois }\end{array}$ & & & 5 \\
\hline -ie & Papousie & & & 1 \\
\hline -ine & $\begin{array}{l}\text { abricotine, cambrésine, indigotine, } \\
\text { myrosine }\end{array}$ & & & 4 \\
\hline -ite & halloysite & & & 1 \\
\hline -al & soucutal & & & 1 \\
\hline -(at)aire & moscoutaire, sursitaire, tissulaire & 1 & 1 & 1 \\
\hline -esque & $\begin{array}{l}\text { alibababesque, cacatesque, } \\
\text { sofalesque... }\end{array}$ & 1 & 1 & 24 \\
\hline -ième & combientième & & & 1 \\
\hline -ien/éen & $\begin{array}{l}\text { sabatéen, hugolien, cambrésien, } \\
\text { onusien }\end{array}$ & 2 & & 3 \\
\hline -if & taxatif & & 1 & \\
\hline -ique & $\begin{array}{l}\text { atlastique, bureautique, hugotique, } \\
\text { sidatique }\end{array}$ & 6 & & 8 \\
\hline -(at)oire & cacatoire & 1 & & \\
\hline -age & $\begin{array}{l}\begin{array}{l}\text { agiotage, émerisage, escargotage, } \\
\text { zieutage }\end{array} \\
\end{array}$ & 6 & 29 & 1 \\
\hline -aille & bijoutaille, bedeaudaille, bleusaille & & & 4 \\
\hline -ée & yeutée & & & 1 \\
\hline -esse & têtutesse & & & 1 \\
\hline
\end{tabular}

4. Le symbole ${ }^{\circ}$ indique la virtualité du terme devant lequel il figure. 
Elaboration d'un corpus morphophonologique : l'épenthèse consonantique

\begin{tabular}{|c|c|c|c|c|}
\hline Suffixe & Illustrations & $\begin{array}{c}\text { Termes } \\
\text { en } \\
\text { balance }\end{array}$ & $\begin{array}{l}\text { Termes } \\
\text { rejetés }\end{array}$ & $\begin{array}{c}\text { Termes } \\
\text { maintenus }\end{array}$ \\
\hline -ille & brindille & & & 1 \\
\hline -(at)ion & $\begin{array}{l}\text { calorisation, catégorisation, } \\
\text { numérotation }\end{array}$ & & 4 & \\
\hline -is & cailloutis & & 1 & \\
\hline -isme & $\begin{array}{l}\text { gagatisme, médiatisme, } \\
\text { inappropriétisme }\end{array}$ & 1 & & 5 \\
\hline -(it)ude & dégourditude & & 1 & \\
\hline -(e)ment & $\begin{array}{l}\text { abritement, bazardement, } \\
\text { gargantualement }\end{array}$ & 2 & 7 & 2 \\
\hline -on & piéton & & 1 & \\
\hline -ot & queusot, gosselot, masselotte & & & 3 \\
\hline -(i)té & cochonceté, conceté, faucuseté & & & 3 \\
\hline -(at)ure & cuirture, noirdure, fermeture, vergeture & & 2 & 3 \\
\hline -(and)ier & $\begin{array}{l}\text { dinandier, chaînetier, abricotier, } \\
\text { morutier }\end{array}$ & 7 & 6 & 36 \\
\hline -ière & $\begin{array}{l}\text { boucautière, cacaotière, cafetière, } \\
\text { lolotière }\end{array}$ & 1 & 1 & 7 \\
\hline -(er)ie & $\begin{array}{l}\text { bijouterie, filouterie, cucuterie, } \\
\text { lamaserie }\end{array}$ & 16 & 1 & 15 \\
\hline -(er)aie & bambouseraie, asergeraie & 1 & & 1 \\
\hline -(at)eur & $\begin{array}{l}\text { émeriseuse, agioteur, noyauteur, } \\
\text { recruteur }\end{array}$ & 2 & 20 & \\
\hline $\begin{array}{l}\text {-(at)eux/- } \\
\text { se }\end{array}$ & crapoteux, cafardeux, caillouteux & 4 & 4 & 5 \\
\hline -ard & vicelard, queutard, banlieusard, faflard & 1 & 1 & 2 \\
\hline -asse & biberondasse & & & 1 \\
\hline $\begin{array}{l}\text {-ar } \\
\text { (finale) }\end{array}$ & escobarderie, canulardesque, bazarder & 9 & & \\
\hline -é & $\begin{array}{l}\text { barreaudé, biseauté, enképissé, } \\
\text { miroité }\end{array}$ & 4 & & 8 \\
\hline -ant & glougloutant, loftstorysant, trotskisant & & 3 & \\
\hline -able & catégorisable & & 1 & \\
\hline -iner & piétiner & & & 1 \\
\hline -iser & abrutiliser, ghettoriser, radiotiser & & 3 & 4 \\
\hline $\begin{array}{l}\text {-iser } \\
\text { (finale) }\end{array}$ & $\begin{array}{l}\text { coloriser, loftstoryser, engéniser, } \\
\text { taniser }\end{array}$ & 8 & & \\
\hline -ir & beausir, glougloutir & & & 2 \\
\hline -er & $\begin{array}{l}\text { adultiner, bisouter, blablater, } \\
\text { bourreauder }\end{array}$ & & 2 & 99 \\
\hline TOTAL & 435 & 73 & 90 & 272 \\
\hline
\end{tabular}




\section{PAGLIANO}

L'ensemble des deux filtres constitue une procédure scientifiquement justifiée, applicable à tout nouveau terme potentiellement porteur d'une épenthèse. Ce protocole a réduit la base de données de 859 termes à 262 (auxquels se sont ajoutés 10 termes virtuels), ce qui signifie que le corpus fini représente moins d'un tiers de l'ensemble des termes initialement rassemblés. Ce n'est cependant qu'à ce prix que les données vont permettre une analyse fiable, tant du point de vue quantitatif que qualitatif, de l'épenthèse consonantique en français entre radical et suffixe.

\section{Exploitation du corpus}

Le corpus comporte maintenant 272 séquences pour lesquelles la validité de l'épenthèse consonantique est bien étayée $^{5}$ : ces dérivés ne sont pas des héritages directs du latin et la consonne présente à la frontière suffixale n'est sous-jacente ni à la base, ni au suffixe. Les coronales sont majoritaires dans ce phénomène d'épenthèse (262 sur 272), particulièrement la coronale [t], qui représente à elle seule plus des trois quarts des épenthèses (205). L'observation de ces termes en fonction de critères phonologiques comme morphologiques met en évidence le manque de pertinence des motivations classiquement proposées à l'épenthèse, comme la longueur du radical ou la position en hiatus.

\subsection{Evaluation des critères morphologiques}

Divers critères morphologiques ont en effet été systématiquement évalués dans cette analyse, à commencer par la nature lexicale des bases et celle des dérivés. Les substantifs déclenchent les trois quarts des dérivés contenant une épenthèse (74.92\%), voire les neuf dixièmes si l'on y ajoute les noms propres (90.9\%). Les verbes en revanche sont fort peu représentés. On ne saurait tirer de conclusion trop hâtive sans une étude approfondie de la structure du lexique construit français. En effet, si les verbes ne sont que très peu utilisés en

5. L'ensemble du corpus et de son traitement figurent dans Pagliano (2003, notamment l'annexe 3c pp.969-1100, disponible également sur http://www.unice.fr/dsl/tobias.htm, rubrique « Other people's papers »). 


\section{Elaboration d'un corpus morphophonologique : l'épenthèse consonantique}

tant que bases pour la dérivation, alors leur quasi-absence de ce corpus n'est que le reflet d'une tendance plus générale et ne revêt aucune signification pour la détermination du contexte de l'épenthèse. La situation est plus homogène si l'on observe les cas d'épenthèse du point de vue de la catégorie lexicale des dérivés et non plus de celle des bases : 120 substantifs contiennent une épenthèse entre radical et suffixe, ce qui est également le cas de 114 verbes et 65 adjectifs.

Le tri en fonction de la taille du radical met en évidence le fait que l'épenthèse n'est pas due à une taille minimale de mot requise par la langue: non seulement les radicaux monosyllabiques ne sont pas les plus représentés (67 termes, soit $24.63 \%$ du corpus : ${ }^{\circ}$ bleutier, noité), mais ceux de trois syllabes et plus sont loin d'être exclus du phénomène de l'épenthèse (44 dérivés, 16.54\% : escargoter, méliméloter). En fait, la majorité du corpus est constitué de termes formés à partir de radicaux bisyllabiques (160 dérivés, soit 58.52\%: 'piapiater, cacatesque).

\subsection{Evaluation des facteurs phonologiques}

Le contexte phonologique, c'est-à-dire la nature des sons en contact immédiat avec le segment épenthésé, a également été étudié : peut-on isoler un type de son particulier qui serait responsable de l'apparition d'une consonne épenthétique?

93.68\% (252 sur 272) des épenthèses ont lieu devant des suffixes dont l'initiale est une voyelle non-arrondie. Cependant, si l'on considère une liste des suffixes français ${ }^{6}$ (par exemple Bechade 1992: 124-132), il est aisé d'établir qu'effectivement, ce sont les suffixes qui manifestent une tendance générale à l'initiale non-arrondie, ce qui signifie qu'il s'agit d'une particularité de la dérivation française et non de l'épenthèse en tant que phénomène phonologique. On peut en outre observer la part des dérivés verbaux dans l'ensemble des dérivés du français : le suffixe verbal -er est présent dans 9982 des 64.296 mots contenus dans la base. Ceci justifie pleinement

\section{Cette liste n'a pas de caractère définitif ni décisif, il s'agit simplement} d'établir une tendance. 


\section{PAgLiano}

la forte représentation de la voyelle initiale de suffixe [e] dans l'ensemble des dérivés du français, en dehors du phénomène de l'épenthèse même ou du fait que relativement peu de suffixes sont à initiale [e].

L'examen du segment radical adjacent au segment épenthésé indique que 29 dérivés (10.66\%) sont formés à partir de bases à finale consonantique. Les voyelles semblent donc majoritaires, particulièrement les voyelles arrondies $(0, \mathrm{u}, \mathrm{y}, \varnothing$, o). On ne peut pourtant considérer qu'il s'agisse là d'un critère déterminant, sans quoi il faudrait expliquer la raison pour laquelle près d'un tiers du corpus (31.28\%) est constitué d'exceptions (i, e, $\varepsilon, \tilde{\varepsilon}$, a, ã). En fait, c'est la voyelle [o] qui se distingue particulièrement des autres par sa fréquence d'occurrence avec les consonnes épenthétiques, plus qu'une classe de voyelles données. Par ailleurs, on constate qu'une voyelle donnée ne sélectionne pas une consonne épenthétique particulière, puisque en dehors de [e] chacune est observée avec plusieurs consonnes épenthétiques. La nature de la consonne, tout comme son existence, ne sont donc pas en rapport avec la nature de la voyelle précédente.

\subsection{Vers une solution mixte : manifestation de la morphologie en phonologie}

La distribution de ces facteurs tant morphologiques que phonologiques semble correspondre à celle de ces mêmes facteurs dans le lexique général de la langue ${ }^{7}$. Aussi ne peut-on attribuer à aucun d'entre eux la responsabilité de l'apparition d'une consonne épenthétique, et doit-on conclure à l'indépendance de l'épenthèse vis-à-vis de la nature mélodique et morphologique des segments environnants. Ceci signifie particulièrement que ni la taille du radical, ni surtout l'hiatus (cf. paragraphe suivant), ne peuvent être considérés comme responsables de l'épenthèse à la dérivation suffixale en français.

Le corpus permet de remettre en question la thèse, courante et intuitive, selon laquelle l'hiatus constitue le motif nécessaire et suffisant à l'apparition d'une épenthèse

7. Cf. Pagliano (2003: 530-541) pour le détail chiffré et exemplifié de l'évaluation de ces différents critères. 

Elaboration d'un corpus morphophonologique : l'épenthèse
consonantique

consonantique. Il n'est pas nécessaire, puisqu'un dixième des épenthèses consonantiques attestées ne se produisent pas en hiatus; il n'est pas suffisant, puisque la langue atteste de nombreux cas d'hiatus non résolus par une épenthèse consonantique. Cette double négation met en évidence l'indépendance de l'hiatus et de l'épenthèse consonantique. Garder l'hiatus comme hypothèse explicative oblige à postuler un deuxième mécanisme déclencheur pour les $10 \%$ des épenthèses (briqueter, atlastique, noirdure, etc.) qui ne se produisent pas en hiatus.

On remarque par ailleurs, grâce au corpus, que tous les suffixes ne déclenchent pas d'épenthèse consonantique; en effet, le corpus final comprend 38 suffixes différents, dont 15 ne concernent qu'un seul dérivé. Il semblerait donc que la nature même du suffixe joue un rôle dans le mécanisme de l'épenthèse, ou plus exactement qu'il existe une classe de suffixes susceptible de se trouver en contact avec une épenthèse consonantique, et qui s'oppose à l'ensemble des suffixes français qui ne sont jamais observés précédés d'une épenthèse.

L'exclusion de l'hiatus comme contexte déclencheur associé à la présence d'une classe particulière de suffixe conduit à proposer une solution unifiée pour l'épenthèse consonantique à la suffixation en français : la frontière morphologique crée les conditions phonologiques nécessaires et suffisantes à l'apparition d'une épenthèse.

Tournons-nous maintenant, non plus vers le contexte, mais vers l'objet lui-même. Phonologiquement parlant, l'épenthèse constitue le renforcement d'une position, au même titre que la gémination consonantique ou dans certains cas la résistance à la lénition (cf. Scheer \& Ségéral 2001). Au sein du cadre théorique CVCV (Lowenstamm 1996), développement récent de la Phonologie de Gouvernement (Kaye \& al. 1985, 1990, Harris 1990) qui se caractérise par une stricte consécution d'attaques (C) et de noyaux (V) au niveau squelettal, une paire de ces positions (CV) constituant l'unité minimale manipulable, et par l'existence de noyaux vides gérés par le Principe des Catégories Vides, les positions consonantiques fortes sont au 
nombre de deux : à l'initiale (\#_V) et après une consonne en "coda" (C._(C)V). Elles s'opposent aux positions faibles (intervocalique, "coda" et consonne finale) en ce qu'elles partagent une propriété structurale : ce sont toutes et seulement les positions non gouvernées mais licenciées, selon le schéma ci-dessous :

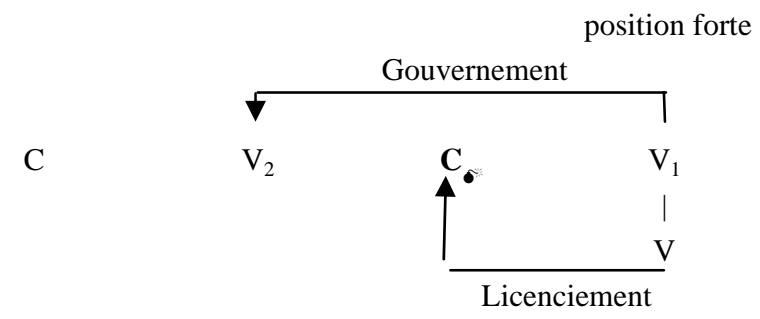

Pour ne pas recevoir de réalisation phonétique, un noyau doit être gouverné par le noyau suivant, qui pour ce faire doit lui-même ne pas être gouverné. Un noyau lexicalement plein est donc en mesure de gouverner un noyau vide précédent ; ici, $V_{1}$ gouverne $V_{2}$. Ce faisant, elle n'est plus en mesure de gouverner sa propre attaque. En revanche, elle licencie celle-ci. C'est la conjonction de l'absence de gouvernement, force qui « inhibe l'expression segmentale de sa cible ", et du licenciement, qui au contraire "conforte l'expression segmentale de sa cible » (Scheer \& Segeral 2001 : 20), qui crée la position forte. Une position forte, parce qu'elle est non gouvernée, est nécessairement précédée d'un noyau vide.

Le gouvernement comme le licenciement sont distribués par la voyelle située à la droite de la position en cause, le contexte gauche étant totalement neutre en regard de sa force ou de sa faiblesse. Cette dernière observation corrobore les données du corpus : une épenthèse peut se produire aussi bien après une consonne (briqueter, cuirture) qu'après une voyelle (bijouter, pouillyzois), pourvu qu'une voyelle lexicalement pleine soit présente à sa droite.

Il peut sembler surprenant de considérer que l'épenthèse en hiatus constitue la résolution d'une position forte, tout 


\section{Elaboration d'un corpus morphophonologique : l'épenthèse consonantique}

d'abord du fait que l'intervocalique est connue pour être une position faible, mais également parce que, comme je l'ai établi plus haut, un noyau vide doit précéder la position pour que celle-ci ne reçoive pas de gouvernement, et donc soit forte dans le cadre théorique considéré. Ceci indique donc qu'au niveau sous-jacent, la situation n'est pas celle qu'il paraît à la surface : il doit y avoir une position vocalique vide - donc une unité CV dans le cadre théorique choisi - présente entre les deux voyelles lexicalement pleines. D'où peut provenir cette position vide ?

J'ai établi plus haut le lien entre un certain type de frontière morphologique et l'épenthèse : la frontière précédant certains suffixes crée les conditions nécessaires à l'apparition d'une épenthèse. Je propose donc que la frontière morphologique située devant ces suffixes se manifeste au niveau phonologique sous la forme d'une unité CV lexicalement vide. Cette traduction d'une propriété morphologique en une unité représentationnelle phonologique n'est pas nouvelle, mais s'inscrit dans un mouvement plus général. Lowenstamm (1999) a ainsi proposé d'identifier la frontière de début de mot par une unité CV. Depuis quelques années en effet, les linguistes travaillant en Phonologie de Gouvernement se sont attelés à la tâche de proposer une identité phonologique des objets morphologiques, de façon à ce que la phonologie travaille non avec des diacritiques (\#, +, etc.) mais avec des éléments phonologiques (cf. par exemple Lahrouchi 2001, Rucart 2003, Scheer sous presse).

Le français distingue donc a minima deux classes de suffixes ${ }^{8}$, ce que j'ai établi plus haut et qui correspond à ce que Siegel (1974) et la Phonologie Lexicale ont établi pour les suffixes anglais. Il est maintenant possible de gratifier ces deux classes de suffixes d'une identité phonologique: ceux qui peuvent s'observer précédés d'une épenthèse, comme -er, se voient attribuer par la morphologie une unité CV à la frontière morphologique; ceux qui ne sont jamais précédés d'une épenthèse, par exemple -âtre, sont précédés d'une frontière

\section{8. $C f$. listes en appendice.}




\section{PAGLIANO}

morphologique qui ne se manifeste pas sous la forme d'une unité $\mathrm{CV}$.

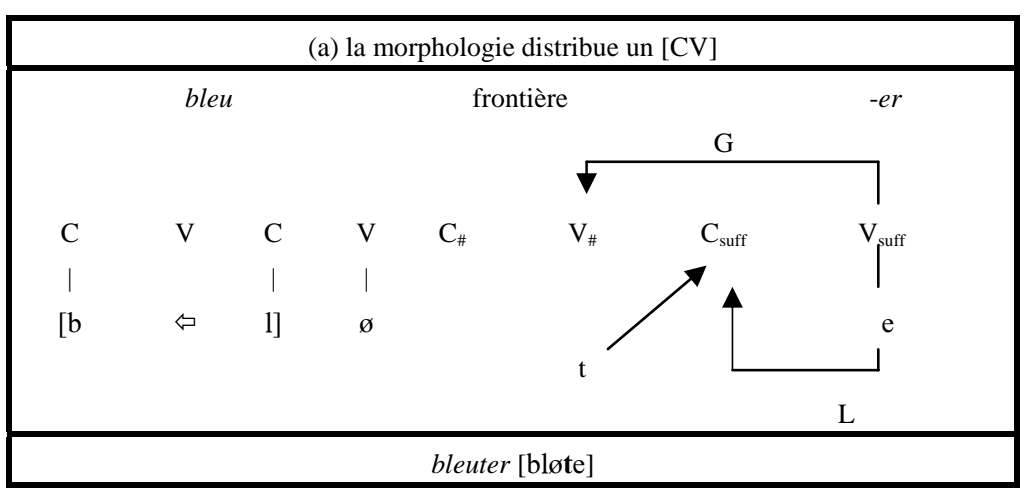

\begin{tabular}{|c|c|c|c|c|c|c|c|c|c|}
\hline \multicolumn{10}{|c|}{ (b) la morphologie ne distribue pas de [CV] } \\
\hline \multicolumn{4}{|c|}{ bleu } & \multicolumn{6}{|c|}{-âtre } \\
\hline $\mathrm{C}$ & $\mathrm{V}$ & $\mathrm{C}$ & $\mathrm{V}$ & $\mathrm{C}_{\text {suff }}$ & $\mathrm{V}_{\text {suff }}$ & $\mathrm{C}$ & $\mathrm{V}$ & $\mathrm{C}$ & $\mathrm{V}$ \\
\hline | & & | & | & & | & | & & | & \\
\hline$[\mathrm{b}$ & ڤ & 1] & $\varnothing$ & & $\mathrm{a}$ & {$[\mathrm{t}$} & ڤ & $\chi]$ & \\
\hline & & & & bleuât & løatr & & & & \\
\hline
\end{tabular}

Lorsque la morphologie distribue une unité CV, comme dans le cas de bleuter, c'est la présence d'un noyau vide avant la position consonantique qui crée les conditions nécessaires à sa force, lui permettant de recevoir l'épenthèse consonantique. Dans le cas de bleuâtre en revanche, la morphologie ne distribuant pas d'unité $\mathrm{CV}$, la séquence ne comporte aucune position forte susceptible de se voir attribuer une épenthèse.

Cette analyse conduit à prendre position quant à la représentation sous-jacente des suffixes qui pourraient être considérés en tant que suffixes à initiale consonantique (-ier à initiale [j] dans table-t-ier, -(e)raie à initiale [ь] dans bambou-s-eraie) présents dans le corpus: 76 dérivés sont 


\section{Elaboration d'un corpus morphophonologique : l'épenthèse consonantique}

concernés, soit $27.57 \%$. En effet, la force d'une position est directement liée à la présence d'un noyau lexicalement plein à sa droite, et non d'une consonne : aucune épenthèse ne peut avoir lieu devant un suffixe à initiale consonantique. Deux cas se distinguent : soit le suffixe débute par une semi-voyelle (-ier, -ième, -ien, -ois), soit non (-elet, -ement, -eraie, -erie, -eté).

Dans le premier cas, le glide constitue le premier élément d'un noyau branchant, qui gouverne la position vocalique apportée par la frontière morphologique. En français coexisteraient alors deux suffixes -ier : l'un comporte le glide en noyau, ce qui permet à une consonne flottante de s'associer (grossier) ou à une consonne d'être épenthésée (tabletier) ; dans l'autre le glide est syllabifié en attaque, ce qui lui permet de se propager sur le noyau précédent (tablier). Une unité [CV] est insérée avant le premier suffixe, ce qui se manifeste par l'épenthèse; pour le deuxième suffixe, rien ne permet de déterminer si la morphologie distribue ou non une unité [CV].

Dans le cas des suffixes de type -elet, la consonne «initiale» de suffixe est en réalité précédée d'un schwa lexical $^{9}$, si bien que la représentation contient un noyau en mesure de gouverner la position vocalique supplémentaire.

A partir du moment où l'hiatus est rejeté en tant que contexte déclencheur de l'épenthèse en français à la frontière suffixale, il est possible de mettre en relation ce type d'épenthèse avec les autres cas d'épenthèses consonantiques en français : à l'emphase avec l'épenthèse d'une occlusive glottale (c'est impossible [sct?z̃posibl]) et entre un verbe et un clitique $\left(a-t-i l\right.$ [atil], parles-en [parlzã]] ${ }^{10}$. En effet, ces deux cas d'épenthèse se produisent également après consonne. Cette unification des trois cas d'épenthèses consonantiques en français n'est possible que parce que le corpus a mis en évidence l'absence de lien nécessaire entre l'hiatus et l'épenthèse.

9. Cette question est marginale en regard de l'objectif poursuivi par le présent article, cf. Pagliano (2003: 784-802) pour l'analyse complète des suffixes à initiale « consonantique ».

10. Pour une analyse circonstanciée mettant en évidence le caractère épenthétique de ces consonnes, $c f$. Pagliano (2003 : 614-727). 


\section{PAGLIANO}

\section{Conclusion}

Le bilan de cet article se présente sous deux aspects : l'un, formel, concerne les méthodes d'élaboration d'un corpus morphophonologique; l'autre souligne l'intérêt d'un corpus raisonné dans l'étude de phénomènes phonologiques en général, et concernant l'épenthèse consonantique en particulier.

\subsection{Bilan des différentes méthodes exploitées dans la constitution du corpus}

Au terme du processus de filtrage qui a réduit le corpus de 859 à 262 séquences peut être établi un bilan en matière d'efficacité et de rentabilité des différentes méthodes de recueil de termes. Le tableau ci-dessous permet d'évaluer la représentation de chacune des sources dans le corpus, en regard de son poids dans la base de données initiale :

\begin{tabular}{|c|c|c|c|c|c|}
\hline type de source & 惹 & 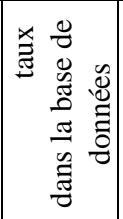 & 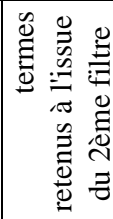 & 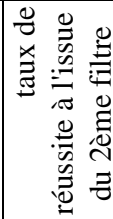 & 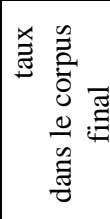 \\
\hline liste de 64.296 termes & 21 & $2.44 \%$ & 5 & $23.81 \%$ & $1.91 \%$ \\
\hline dictionnaires d'argot & 64 & $7.45 \%$ & 23 & $35.94 \%$ & $8.78 \%$ \\
\hline interrogation du TLFi & 48 & $5.59 \%$ & 26 & $54.17 \%$ & $9.92 \%$ \\
\hline articles spécialisés ou profanes & 94 & $10.95 \%$ & 47 & $50 \%$ & $17.94 \%$ \\
\hline $\begin{array}{l}\text { étude des suffixes -ier, -age et } \\
\text {-erie }\end{array}$ & 350 & $40.74 \%$ & 66 & $18.86 \%$ & $25.19 \%$ \\
\hline dictionnaire des néologismes & 25 & $2.91 \%$ & 12 & $48 \%$ & $4.58 \%$ \\
\hline dictionnaire des mots sauvages & 61 & $7.10 \%$ & 20 & $32.79 \%$ & $7.63 \%$ \\
\hline total sources écrites & 663 & $77.18 \%$ & 199 & $30.02 \%$ & $75.95 \%$ \\
\hline sources orales & 196 & $22.82 \%$ & 63 & $32.14 \%$ & $24.05 \%$ \\
\hline total du corpus & 859 & $100 \%$ & 262 & $30.50 \%$ & $100 \%$ \\
\hline
\end{tabular}

Moins d'un tiers des termes présélectionnés ont été retenus dans le corpus (30.50\%), ce qui s'explique par le manque de rentabilité de certains des moyens utilisés. La 


\section{Elaboration d'un corpus morphophonologique : l'épenthèse consonantique}

méthode la moins intéressante se révèle être le tri par un suffixe précis (moins de 19\% de maintien), suivie par l'exploitation semi-automatique de la base de données (moins d'un quart). Ces deux méthodes ont pour point commun leur source - la liste de 64.296 mots - ainsi qu'une certaine automaticité de la récolte. Soit cette liste contient peu de termes intéressants du fait qu'il s'agit d'un dictionnaire général, au contraire des autres dictionnaires exploités; soit la méthode d'extraction est perfectible. En ce qui concerne cette dernière, on retiendra cependant un certain nombre de critères de préparation des mots, tels l'élision des préfixes, le traitement des accents et de certaines suites graphiques (-qu-, -eux, etc.). Seront également à prendre en compte dans tout travail futur les difficultés relatives aux alternances de radicaux, de suffixes, à l'identification de la frontière morphologique et à la graphie elle-même.

Les dictionnaires se sont en revanche révélés très rentables: $35.94 \%$ de termes retenus pour les dictionnaires d'argot, $32.79 \%$ pour le Dictionnaire des mots sauvages, et surtout $48 \%$ pour le Dictionnaire des néologismes. L'interrogation du TLFi par mots-clés s'est avérée fructueuse : plus de la moitié des termes sélectionnés se retrouvent dans le corpus. Quant aux articles, 50\% des termes ont été maintenus.

Si l'on observe de plus près l'apport effectif de chacune des sources exploitées dans la constitution de ce corpus, non plus en termes de rentabilité mais en termes quantitatifs simplement, le traitement automatique de la liste de 64.296 mots se révèle encore une fois déficitaire puisque son apport représente moins de $2 \%$ du corpus total. Par ailleurs, les méthodes les plus rentables ne sont pas celles qui au total ont fourni le plus de termes au résultat final, puisque l'interrogation du TLFi comme l'exploitation du dictionnaire des néologismes ont chacune apporté moins de $10 \%$ des termes présents dans le corpus. En ce qui concerne le corpus écrit, ce sont les articles qui ont le plus contribué au corpus puisque près d'un terme sur cinq provient de cette source. 


\section{PAgLiano}

\subsection{Bilan sur le rôle du corpus dans la recherche sur l'étude des suffixes}

La constitution rigoureuse d'un corpus raisonné portant sur l'épenthèse consonantique à la frontière dérivationnelle suffixale en français a permis non seulement de rejeter les hypothèses «classiques » de taille minimale de dérivé ou de résolution d'hiatus, mais également de mettre en évidence le lien entre l'épenthèse et certains suffixes.

C'est cette double constatation qui a conduit à une solution prenant en compte l'ensemble des données du corpus, à savoir les épenthèses apparaissant en hiatus comme celles se produisant après une consonne. L'hypothèse formulée a cet avantage supplémentaire d'unifier l'ensemble des cas d'épenthèses consonantiques en français - à l'emphase, entre verbe et clitique, à la dérivation suffixale - sous une même dimension explicative : la résolution d'une position forte.

\subsection{Sur le va-et-vient entre théorie et empirie}

Un dernier point reste à souligner : l'impact réciproque du corpus et de l'analyse. En effet, la problématique de l'épenthèse consonantique à la frontière suffixale est née pour moi de l'analyse des adverbes en -ment en français ( $c f$. Pagliano 1999) qui avait conclu à la concomitance de la frontière suffixale devant l'adverbe avec une unité [CV], indépendamment de l'épenthèse. La question de l'identité de cette unité [CV] restait alors en suspens : appartenait-elle au morphème suffixal ou était-elle la manifestation phonologique de la frontière morphologique ? L'idée était alors la suivante : si l'unité $[\mathrm{CV}]$ correspond à la frontière suffixale, elle doit également se rencontrer avec d'autres suffixes. Quel critère pouvait permettre de mettre en évidence cette frontière ? Si la frontière se traduit par l'ajout d'une unité [CV], je dois être en mesure d'observer la manifestation de cette unité au moins dans certains contextes. Puisqu'il s'agit d'un ajout, les épenthèses situées précisément à cette frontière semblent constituer un indice révélateur de la concrétisation de la frontière par l'unité [CV] supposée. C'est donc bien la théorie qui a conduit à l'élaboration de l'empirie concernant l'épenthèse consonantique. 


\section{Elaboration d'un corpus morphophonologique : l'épenthèse consonantique}

A l'inverse, c'est la mise en place d'un corpus selon des critères précis, et non le rassemblement aléatoire de données brutes, qui a permis d'établir et d'asseoir le rejet de l'hiatus comme contexte nécessaire et suffisant à l'apparition d'une épenthèse consonantique.

\section{Références bibliographiques}

Apothéloz D. (2002). La construction du lexique français. Gap / Paris : Ophrys (Collection « L'essentiel »).

Bechade H. D. (1992). Phonétique et morphologie du français moderne et contemporain. Paris : PUF.

Bloch O. \& von Wartburg W. (19685). Dictionnaire étymologique de la langue française. Paris : PUF.

Caradec F. (2000). Dictionnaire du français argotique et populaire. Paris : Larousse.

Cellard J. \& A. Rey (1980). Dictionnaire du français nonconventionnel. Paris : Hachette.

Colin J.-P. et al. (1990 [2001]). Dictionnaire de l'argot français et de ses origines. Paris : Larousse.

Corbin D. (1987 [1991]). Morphologie dérivationnelle et structuration du lexique, Tomes 1 et 2. Lille: Presses Universitaires de Lille (Collection Sens \& Structures.).

Dubois J. \& Dubois-Charlier F. (1999). La dérivation suffixale en français. Paris : Nathan Université.

George K.E.M. (2000). Néologismes du français contemporain. Datations et documents lexicologiques. Paris : Klincksieck.

Gilbert P. (1980). Dictionnaire des mots contemporains. Paris : Les usuels du Robert (Robert Langage Guides).

Harris J. (1990). "Segmental complexity and phonological government », Phonology 7 : 255-300.

Kaye J. \& al. (1985). "The internal structure of phonological elements: a theory of charm and government ", Phonology Yearbook 2 : 305-328.

Kaye J. \& al. (1990). « Constituent structure and government in phonology », Phonology 7 : 193-231. 


\section{PAGLIANO}

Lahrouchi M. (2003). « La structure des racines en berbère », communication, Colloque du GDR Phonologie, Montpellier.

Littré P.-E. (1863-1877). Dictionnaire de la langue française, 5 volumes. Versailles : Encyclopaedia Britannica France.

Lowenstamm J. (1996). " CV as the only syllable type ", in J. Durand \& B. Laks (eds.), Current Trends in Phonology, Models and Methods, pp. 419-441.

Lowenstamm J. (1999). "The beginning of the word", Syllables!? : 153-166.

Pagliano C. (1999). Formation des adverbes en -ment en français. Mémoire de DEA, Université de Nice SophiaAntipolis.

Pagliano C. (2003). L'épenthèse consonantique en français. Ce que la syntaxe, la sémantique et la morphologie peuvent faire à la phonologie: parles-en de ta numérotation Pimpossible. Thèse de doctorat, Université de Nice SophiaAntipolis.

Rey A. \& al. (1992-1998 [2001]). Robert, Dictionnaire historique de la langue française. Paris : Dictionnaire Le Robert.

Rey-Debove J. \& Gagnon G. (1981). Dictionnaire des anglicismes : les mots anglais et américains en français. Paris : Robert (Collection Les Usuels du Robert).

Rheims M. (1989). L'Insolite, Dictionnaire des mots sauvages des écrivains des 19 e et 20e siècles. Paris : Larousse.

Rucart P. (2003). "Ordre des Affixes et Phonologie du Gouvernement en Qafar », communication, Colloque du GDR Phonologie, Montpellier.

Scheer T. \& Ségéral P. (2001). "La coda-miroir », Bulletin de la Société de Linguistique de Paris XCVI : 107-152.

Scheer T. (ms). A Syntagmatic Theory of Phonology.

TLFi (version 2003). Trésor de la Langue Française informatisé, CNRS, http://atilf.inalf.fr/tlfv3.htm 
Elaboration d'un corpus morphophonologique : l'épenthèse consonantique

Appendice : deux classes de suffixes en français

\begin{tabular}{|c|c|c|c|}
\hline \multicolumn{2}{|c|}{$\begin{array}{c}\text { Suffixes déclencheurs } \\
\text { potentiels d'épenthèses } \\
\text { (type -er) }\end{array}$} & \multicolumn{2}{|c|}{$\begin{array}{c}\text { Suffixes ne déclenchant } \\
\text { jamais d'épenthèse } \\
\text { (type -âtre) }\end{array}$} \\
\hline -aille & bijoutaille & -age & *balaitage \\
\hline -ain & amalfitais & -aire & *incenditaire \\
\hline -ais & congolais & -al & *idétal \\
\hline -ard & faflard & -asse & *fainétasse \\
\hline -é & abricoté & -eau & *baleineteau \\
\hline -elet & dieutelet & -ée & *chitée \\
\hline -ement & piètement & -esse & *harditesse \\
\hline -er & abriter & -ette & *balaitette \\
\hline -erie & ferblanterie & -ie & *accalmetie \\
\hline -esque & cacatesque & -ite & *jésutite \\
\hline -eté & coconceté & -on & *dindeton \\
\hline -eux & caillouteux & & \\
\hline -ien & cambrésien & & \\
\hline -ier & coquetier & & \\
\hline -in & piétin & & \\
\hline -ine & abricotine & & \\
\hline -ique & atlastique & & \\
\hline -ir & glougloutir & & \\
\hline -iser & radiotiser & & \\
\hline -isme & gagatisme & & \\
\hline -ois & spadois & & \\
\hline -ot & gosselot & & \\
\hline -ure & cuirture & & \\
\hline
\end{tabular}

